\title{
Interaction of soyasaponins with plant ingredients in diets for Atlantic salmon, Salmo salar L.
}

\author{
Elvis M. Chikwati ${ }^{1 *}$, Fredrik F. Venold ${ }^{1}$, Michael H. Penn ${ }^{1}$, Jens Rohloff ${ }^{2}$, Ståle Refstie ${ }^{1,3}$, Arne Guttvik ${ }^{4}$, \\ Marie Hillestad ${ }^{4}$ and Åshild Krogdahl ${ }^{1}$ \\ ${ }^{1}$ Department of Basic Sciences and Aquatic Medicine, Norwegian School of Veterinary Science, Aquaculture Protein Centre \\ (a CoE), PO Box 8146 Dep, NO-O033 Oslo, Norway \\ ${ }^{2}$ The Plant Biocentre, Department of Biology, Norwegian University of Science and Technology (NTNU), 7471 Trondheim, \\ Norway \\ ${ }^{3}$ Aquaculture Protein Centre (a CoE), Nofima Marine, 6600 Sunndalsøra, Norway \\ ${ }^{4}$ Biomar AS, Nordre Gate 11, 7011 Trondheim, Norway
}

(Submitted 21 March 2011 - Final revision received 18 July 2011 - Accepted 2 August 2011 - First published online 14 September 2011)

\begin{abstract}
The effects of combining soyasaponins with plant ingredients on intestinal function and fish health were investigated in an $80 \mathrm{~d}$ study with Atlantic salmon $(270 \mathrm{~g})$ distributed thirty each into twenty-four tanks with seawater. Soyasaponins were supplemented (2 g/kg) to diets with maize gluten (MG), pea protein concentrate (PPC) and sunflower (SFM), rapeseed (RSM) or horsebean meals. A diet with soyabean meal (SBM) and another with wheat gluten and soyasaponins served as reference diets. Marked soyasaponin effects were observed when combined with PPC. This combination induced inflammation in the distal intestine (DI) similar to SBM, reduced feed intake, apparent digestibility of lipid, most amino acids and ash, decreased bile salt levels in intestinal chyme and decreased leucine aminopeptidase (LAP) activity but increased trypsin activity in the DI. No enteritis was observed in other diet groups, but small consistent negative soyasaponin effects were seen on lipid and fatty acid digestibility, faecal DM and LAP activity of the DI. Soyasaponin combination with RSM reduced digestibility of all nutrients including minerals. The mineral effect was also seen for SFM, whereas with MG and SFM a positive soyasaponin effect on feed intake was observed. Caution should be exercised to avoid ingredient combinations giving high saponin levels, a condition that appears to be a key factor in diet-induced enteritis together with certain plant ingredients.
\end{abstract}

Key words: Soyasaponins: Plant protein ingredients: Antinutritional factors: Fish feed: Gastrointestinal tract

Alternative dietary protein sources to supplement and replace limited marine ingredients in fish feeds are important for the future of the fish farming industry. Plant ingredients such as soyabeans hold promise with their good amino acid profiles that can easily be improved for fish requirements by supplementation with deficient amino acids. However, soyabean meal (SBM) inclusion has been demonstrated to induce enteritis and reduce performance in salmonids ${ }^{(1-3)}$ and carp ${ }^{(4)}$. The factors responsible for the disorders have not been conclusively identified, but soyasaponins, and possibly other bioactive antinutritional factors (ANF) in SBM, are implicated in the aetiology ${ }^{(5-7)}$.

Saponins are heat-stable glycosides present in soyabean and other legumes such as pea and lupin ${ }^{(8,9)}$. Saponins, with their membrane-active nature and affinity to cholesterol and bile salts ${ }^{(10,11)}$, possess a number of potential biological effects compatible with the negative effects observed in fish fed diets containing SBM. There have been conflicting findings from studies on dietary effects of saponins to teleost fish. In one study, a saponin-rich extract from SBM and Quillaja saponins, both at a $0.3 \%$ dietary inclusion rate of saponin, greatly reduced feed intake and growth in Chinook salmon and depressed growth in rainbow trout ${ }^{(12)}$. Furthermore, the Quillaja saponin diets $(0.15$ and $0.3 \%$ dietary inclusion) both induced substantial damage to the intestinal mucosa for both Chinook salmon and rainbow trout. On the other hand, Francis et al. ${ }^{(13)}$ concluded that saponins from Quillaja

Abbreviations: AD, apparent digestibility; ANF, antinutritional factors; BBM, brush-border membrane; CP, crude protein; DI, distal intestine; EAA, essential amino acids; FDM, faecal DM; FER, feed efficiency ratio; GIT, gastrointestinal tract; HB, horsebean; HBM, horsebean meal; LAP, leucine aminopeptidase; MG, maize gluten; MI, mid-intestine; NEAA, non-essential amino acids; OSI, organo-somatic index; PI, pyloric intestine; PP, pea protein; PPC, pea protein concentrate; RS, rapeseed; RSM, rapeseed meal; SBM, soyabean meal; SFM, sunflower meal; ST, stomach; TGC, Thermal-unit growth coefficient; WG, wheat gluten.

*Corresponding author: E. M. Chikwati, fax +47225973 10, email elvis.chikwati@nvh.no 
at similar dietary inclusion levels as previously indicated did not affect feed intake, but rather induced better feed conversion efficiency and growth in common carp (Cyprinus carpio L.) and Nile tilapia (Oreochromis niloticus L.). More recently, support has been strengthened for the involvement of saponins in the distal intestinal enteritis induced by SBM in Atlantic salmon; similar morphological changes were induced in Atlantic salmon when soyasaponins were added to a lupin kernel basal diet at inclusion levels of 0.17 and $0 \cdot 26 \%^{(6,7)}$.

Findings from feeding salmonids diets with SBM have set a precedent in the approach to the evaluation of new plant ingredients for use as alternative protein sources in fish feeds. The present study was designed to investigate the impact of soyasaponins on fish performance and physiology and to find possible interactions between soyasaponins and current plant protein source, ingredients that, to some extent, have been investigated earlier as ingredients in diets for Atlantic salmon ${ }^{(14-16)}$

\section{Materials and methods}

\section{Diets}

Effects of adding soyasaponin to maize gluten (MG; Zea mays L.), pea protein concentrate (PPC; Lathyrus aphaca), sunflower meal (SFM, Helianthus anuus), rapeseed meal (RSM, Brassica napus) or horsebean meal (HBM, Vicia faba var. equina) as dietary plant protein source were investigated. The five protein sources were included at levels as high as practically possible in commercial diet formulations, taking the varying fibre content into consideration, with standardisation regarding protein replacement and protein:energy ratio. As the fibre and protein content of the selected sources differed greatly, two levels of dietary protein replacement were used; MG and PPC were included at a level corresponding to $33 \%$ of total protein, while SFM, RSM and HBM were at $21 \%$. The diets were formulated to contain an equal crude protein (CP):energy ratio of $20 \mathrm{~g} / \mathrm{MJ}$. The strategy was to let dietary energy levels vary to avoid using fillers in the diets that may influence results. Each of these five protein sources was investigated without and with soyasaponin supplementation $(0 \cdot 2 \%)$. Hereafter, the term saponin will refer to soyasaponin regarding the supplementation in the present study. The supplementation level approximated a level provided by a $20-30 \%$ SBM dietary inclusion. In addition, two reference diets were made, one with wheat gluten (WG, Triticum spp.) at an inclusion corresponding to $33 \%$ of total protein and supplemented with saponins, and a second with SBM (Glycine max) included at $21 \%$ of total protein, a level known to cause clear enteritis but usually without severe depression of feed intake.

In all of the diets, protein from the various plant sources partially replaced marine fish protein derived from a combination of Nordic LT (Norsildmel AS, Bergen, Norway) and South American Superprime fishmeals. All diets were supplemented with standard vitamin and micromineral premixes and contained $100 \mathrm{mg} / \mathrm{kg}$ yttrium oxide as an inert marker for the calculation of nutrient apparent digestibilities (AD). The diets were produced by extrusion at the BioMar Feed Technology Centre (Brande, Denmark) with a pellet size of $5 \mathrm{~mm}$ in batches of $50 \mathrm{~kg}$. Detailed diet formulations are shown in Table 1 and chemical composition, as analysed, is shown in Tables 2 and 3.

\section{Experimental animals and conditions}

The present experiment was conducted in compliance with laws regulating experimentation with live animals in Norway as overseen by the Norwegian Animal Research Authority (Forsøksdyrutvalget). The feeding trial was conducted at the land-based Nofima Marin research station at Sunndalsøra, Norway. Atlantic salmon (Salmo salar L.) post smolts of the Sunndalsøra breed with mean weight of $270 \mathrm{~g} \pm 10 \%$ were allocated, thirty each, into twenty-four, $1 \mathrm{~m}^{3}$ fibreglass tanks with 500 litres of saltwater flowing at a rate of 20 litres $/ \mathrm{min}$. Water temperature varied between 9 and $13^{\circ} \mathrm{C}$. The oxygen content and salinity of the outlet water were monitored. Salinity ranged between 31.4 and 33.3 with an average of $32.4 \mathrm{~g} / \mathrm{l}$. A $24 \mathrm{~h}$ lighting regimen was employed during the experimental period.

The fish were fed to satiation using automatic disc feeders refilled every $3 \mathrm{~d}$ with weighed amounts; waste feed was collected daily, separated from faeces, weighed and stored at $-20^{\circ} \mathrm{C}$. Every $3 \mathrm{~d}$, the waste feed level and the percentage recovery of DM from each diet were used to determine the approximate feed intake for each tank. The feed intake for each tank was in turn used to adjust the feeding level every $3 \mathrm{~d}$ to provide at least $20 \%$ excess feed per d. Before starting the experiment, all fish per tank were weighed in bulk.

\section{Sampling}

At the start of the feeding period, twenty fish from the same group as the fish in the experiment were sampled, euthanised by a lethal dose of tricaine methane-sulfonate (MS222; Argent Chemical Laboratories, Inc., Redmond, WA, USA), individually weighed and frozen for whole body composition analysis.

The feeding trial ran for $80 \mathrm{~d}$ with an intermediate sampling (day 30) and a terminal sampling (day 80). At both samplings, tank order and fish sampling were conducted randomly. All sampled fish were euthanised by overdosing with tricaine methane-sulfonate (MS222) and weight and length recorded.

On day 30, the fish were weighed in bulk and three fish from each tank were weighed individually and sampled for histology. The fish were dissected and the gastrointestinal tract (GIT) removed and cleaned of associated adipose tissue. Liver, and mid (MI) and distal intestines (DI) were weighed to calculate organo-somatic indices (OSI). Histology samples were taken from the pyloric, mid and distal segments of the intestine and the liver, fixed in phosphate-buffered formalin ( $4 \%$ formaldehyde) for $24 \mathrm{~h}$ and then transferred to ethanol (70\%).

At termination of the experiment (day 80), twelve fish from each tank were randomly selected and euthanised as 
Table 1. Formulation of the experimental diets $^{\star}$

\begin{tabular}{|c|c|c|c|c|c|c|c|c|c|c|c|c|}
\hline \multirow[b]{2}{*}{ Ingredients } & \multicolumn{12}{|c|}{ Diets (\%) } \\
\hline & MG-0 & MG-S & PP-0 & PP-S & SF-0 & SF-S & RS-0 & RS-S & HB-0 & HB-S & WG-S & SBM \\
\hline Nordic LT-meal† & $23 \cdot 1$ & $23 \cdot 1$ & 21.5 & 21.5 & $26 \cdot 2$ & $26 \cdot 2$ & $24 \cdot 8$ & $24 \cdot 8$ & 24.5 & 24.5 & 23.9 & $26 \cdot 5$ \\
\hline Superprime fish meal $\ddagger$ & 23.1 & $23 \cdot 1$ & 21.5 & 21.5 & $26 \cdot 2$ & $26 \cdot 2$ & 24.8 & 24.8 & 24.5 & 24.5 & 23.9 & $26 \cdot 5$ \\
\hline WG§ & - & - & - & - & - & - & - & - & - & - & $21 \cdot 0$ & - \\
\hline MG\| & $26 \cdot 0$ & $26 \cdot 0$ & - & - & - & - & - & - & - & - & - & - \\
\hline PPCף & - & - & $31 \cdot 0$ & $31 \cdot 0$ & - & - & - & - & - & - & - & - \\
\hline HP soya** & _- & _- & - & - & - & - & - & - & _- & - & _- & $20 \cdot 0$ \\
\hline HP sunflowert† & - & - & - & - & 23.0 & 23.0 & - & - & - & - & - & - \\
\hline 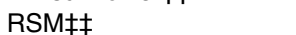 & _ & - & _- & _- & - & - & $27 \cdot 0$ & $27 \cdot 0$ & - & _- & _- & _- \\
\hline $\mathrm{HB}^{\S \S}$ & - & - & - & - & - & - & - & - & $34 \cdot 6$ & 34.6 & - & - \\
\hline Saponins \|\| & - & 0.2 & - & 0.2 & - & 0.2 & - & 0.2 & - & 0.2 & 0.2 & - \\
\hline Tapiokaๆ ๆ & $6 \cdot 0$ & $6 \cdot 0$ & $6 \cdot 0$ & $6 \cdot 0$ & $6 \cdot 0$ & $6 \cdot 0$ & $6 \cdot 0$ & $6 \cdot 0$ & 0.0 & 0.0 & 6.0 & $6 \cdot 0$ \\
\hline Fish oil & $11 \cdot 8$ & $11 \cdot 8$ & $10 \cdot 7$ & $10 \cdot 7$ & $10 \cdot 4$ & $10 \cdot 4$ & 9.9 & 9.9 & $9 \cdot 6$ & $9 \cdot 6$ & $12 \cdot 9$ & $11 \cdot 3$ \\
\hline RS oil & $11 \cdot 8$ & $11 \cdot 8$ & $10 \cdot 7$ & $10 \cdot 7$ & $10 \cdot 4$ & $10 \cdot 4$ & $9 \cdot 9$ & 9.9 & 9.6 & $9 \cdot 6$ & $12 \cdot 9$ & $11 \cdot 3$ \\
\hline Vitamin-mineral-mix ${ }^{\star \star *}$ & 0.38 & 0.38 & 0.38 & 0.38 & 0.38 & 0.38 & 0.38 & 0.38 & 0.38 & 0.38 & 0.38 & 0.38 \\
\hline Lys & 0.21 & 0.21 & - & - & - & - & - & - & - & - & 0.13 & - \\
\hline DL-Met & - & - & 0.37 & 0.37 & - & - & - & - & - & - & - & - \\
\hline Carophyll pink & 0.04 & 0.04 & 0.04 & 0.04 & 0.04 & 0.04 & 0.04 & 0.04 & 0.04 & 0.04 & 0.04 & 0.04 \\
\hline Monocalcium phosphate & 0.30 & 0.30 & 0.51 & 0.51 & - & - & - & - & - & - & 0.30 & - \\
\hline
\end{tabular}

MG, maize gluten, -0, non-inclusion; -S, inclusion; PP, pea protein; SF, sunflower; RS, rapeseed; HB, horsebean; WG, wheat gluten; SBM, soyabean meal; PPC, PP concentrate; RSM, RS meal.

* The plant ingredients were included at levels about as high as possible taking into account bulkiness and expected effects on feed intake and fish performance decided based on earlier experiences. The low-fibre plant ingredients - MG, PPC and WG - were included at levels corresponding to $33 \%$ of total crude protein, whereas the highfibre plant ingredients - SF meal, RSM, HB meam and SBM - were included at levels corresponding to $21 \%$ of total protein.

† Nordic LT 94 fishmeal supplied by Norsildmel AS, Bergen, Norway.

‡ Superprime fishmeal supplied by Köster Marine Proteins GmbH, Hamburg, Germany.

$\S$ WG supplied by Roquette (Beinheim, France).

\| MG supplied by Cargil Nordic (SAS van Gent, Holland).

I PPC made from yellow peas by air classification; supplied by AgriMarin Nutrition (Stavanger, Norway).

** SBM supplied by Scanmills AS (Kolding, Denmark).

†† Heat-treated and hexane-extracted SF meal; supplied by DLA Agro (Galten, Denmark).

㧊 Heat-treated RSM supplied by Emmelev Mølle (Otterup, Denmark).

$\S \S$ Whole horsebeans supplied by Overgaard Gods (Havndal, Denmark).

III The $95 \%$ soyasaponin extract from soyabeans (Glycine max) supplied by Organic Technologies (Ohio, USA).

ๆ ๆ Tapioca supplied by KMC, Brande, Denmark.

*** Supplied to ensure that the diets cover requirements for vitamins and minerals.

described earlier. From six fish, blood was collected in heparinised vacutainers for plasma preparation. From these same fish, the GIT was removed. Intestinal contents from the stomach (ST), cranial and caudal halves of the pyloric intestine (PI1 and PI2), MI and the cranial and caudal halves of the DI (DI1 and DI2) were collected into pre-weighed tubes. Tubes were subsequently frozen in liquid $\mathrm{N}_{2}$ and stored at $-80^{\circ} \mathrm{C}$ for bile salt and trypsin analyses. The organs were put back in place; the fish were re-weighed, individually packed into plastic bags and frozen at $-20^{\circ} \mathrm{C}$ for whole body analyses. From the other six fish, the GIT was removed and freed of associated adipose tissue before histology samples were collected from the cardiac (ST1) and pyloric ST (ST2), PI, MI, DI, liver, spleen, head kidney, trunk (urinary) kidney, gills and muscle, and fixed in neutral buffered formalin for $24 \mathrm{~h}$ and then transferred into $70 \%$ ethanol. The liver was weighed before the histological samples were collected. The remaining MI and DI tissues were collected into pre-weighed containers, frozen in liquid $\mathrm{N}_{2}$ and stored at $-80^{\circ} \mathrm{C}$ for brush-border membrane (BBM) enzyme activity analysis as described previously. Faeces were stripped from the fish remaining in the tanks after sampling for AD measurements. Faeces were stored frozen at $-20^{\circ} \mathrm{C}$ until freeze-drying and analysis. Samples of each diet were collected at start and end of the experiment and stored at $-40^{\circ} \mathrm{C}$ for proximate analysis.

\section{Analyses}

Chemical analyses. Diet and faecal samples were analysed for DM (after heating at $105^{\circ} \mathrm{C}$ for $16-18 \mathrm{~h}$ ), ash (combusted at $550^{\circ} \mathrm{C}$ to constant weight), nitrogen (CP) (by the semi-micro-Kjeldahl method, Kjeltec-Auto System, Tecator, Höganäs, Sweden), fat (diethyl ether extraction in a Fosstec analyser (Tecator, Höganäs, Sweden) after HCl-hydrolysis), starch (measured as glucose after hydrolysis by $\alpha$-amylase (Novo Nordisk A/S, Bagsvaerd, Denmark) and amylo-glucosidase (Bohringer Mannheim GmbH, Mannheim, Germany), followed by glucose determination by the 'Glut-DH method' (Merck, Darmstadt, Germany)), gross energy (using the Parr 1271 Bomb calorimeter, Parr, Moline, IL, USA) and yttrium (by inductivity coupled plasma mass-spectroscopy as described by Refstie $e t a l .{ }^{(17)}$. The amino acids in the diet were analysed using a Biochrom 30 amino acid analyser (Cambridge, UK) following the EC Commission Directive 98/ 64/EC (1999), after hydrolysis in $6 \mathrm{M}-\mathrm{HCl}$ for $23 \mathrm{~h}$ at $110^{\circ} \mathrm{C}$. Tryptophan and tyrosine were analysed after basic hydrolysis.

Plasma variables. Plasma was analysed for NEFA, cholesterol, total TAG and glucose following standard procedures at the Central Laboratory of the Norwegian School of Veterinary Science (NVH), Oslo, Norway.

Trypsin activity and bile salt analyses in intestinal content. Faecal trypsin and bile salt analyses were performed on 
pooled freeze-dried gastrointestinal contents from ST, PI1, PI2, MI, DI1 and DI2.

Trypsin activity was determined colorimetrically, according to Kakade et $a l^{(18)}$, using the substrate benzoyl-arginine$P$-nitroanilide (Sigma no. B-4875; Sigma Chemical Company, St Louis, MO, USA) and a curve derived from a standardised bovine trypsin solution.

Bile salt concentration was determined using the enzyme cycling amplification/Thio-NAD method (Inverness Medical, Cheshire, UK) in the ADVIA $^{\circledR} 1650$ Chemistry System (Siemens Healthcare Diagnostics, Inc., Deerfield, IL, USA) at the Central Laboratory of NVH.

Brush-border membrane enzyme activity analyses. Activity of the BBM enzyme leucine aminopeptidase (LAP; EC 3.4.11.1) was measured in intestinal tissue homogenates. The homogenates were prepared from tissues thawed in ice-cold Tris-mannitol buffer $(1: 20 \mathrm{w} / \mathrm{v})$ containing the serine proteinase inhibitor 4-(2-aminoethyl) benzenesulfonylfluoride $\mathrm{HCl}$ (Pefabloc ${ }^{\circledR}$ SC; Pentapharm Limited, Basel, Switzerland). Activity of LAP was determined colorimetrically using L-leucine- $\beta$-naphthylamide as the substrate as described by Krogdahl et al. ${ }^{(19)}$.

Protein concentration of the homogenates was estimated using the BioRad $^{\circledR}$ Protein Assay (BioRad Laboratories, Munich, Germany). Tissue protein concentration was used in the determination of LAP specific activity.

Intestinal histology. Histology samples were processed according to standard histological techniques and stained with haematoxylin and eosin. The sections were randomised to ensure blinded examination and evaluated using a light microscope. A visual analogue (continuous) scale type scoring system as described by Penn et al. ${ }^{(20)}$ was used to evaluate the intestinal histology. The following tissue characteristics were evaluated: (1) length and fusion (bridging) of the mucosal folds; (2) width and cellularity (leucocyte infiltration, connective tissue hyperplasia) of the lamina propria and submucosa; (3) degree of supranuclear absorptive vacuolisation and nucleus position of enterocytes; (4) frequency of intraepithelial lymphocytes and goblet cells. These are characteristics reported altered in SBM-induced enteritis in Atlantic salmon ${ }^{(2)}$.

\section{Calculations}

$\mathrm{CP}$ was calculated as $\mathrm{N} \times 6 \cdot 25$. Thermal-unit growth coefficient (TGC) was calculated as: $\mathrm{TGC}=\left(\mathrm{FBW}^{1 / 3}-\right.$ $\left.\mathrm{IBW}^{1 / 3}\right) \times\left(\Sigma \mathrm{D}^{\circ}\right)^{-1}$, where IBW and FBW are the initial and final body weights (tank means) and $\Sigma D^{\circ}$ is the thermal sum (feeding days $\times$ average temperature in ${ }^{\circ} \mathrm{C}$ ). Feed intake was estimated by subtracting uneaten (waste) feed from fed feed on a DM basis. The uneaten feed was corrected for DM losses during feeding and collection using estimates of recovery of uneaten feed as described by Helland et $a l .{ }^{(21)}$. Feed efficiency ratio (FER) was calculated as: $G \times F$, where $G$ is the weight gain and $F$ the consumption of DM from the feed. Instantaneous (daily) feed intake (\% of body weight) was estimated as: $\mathrm{FE}=100 \times F_{\mathrm{d}} \times\left(W_{\mathrm{d}-1}+\left(F_{\mathrm{d}-1} \times \mathrm{FER}_{\mathrm{P}}\right)\right)$, where $F_{\mathrm{d}}$ represents feed intake at day, $W_{\mathrm{d}-1}$ and $F_{\mathrm{d}-1}$ are weight and feed intake the previous day and $\mathrm{FER}_{\mathrm{P}}$ is

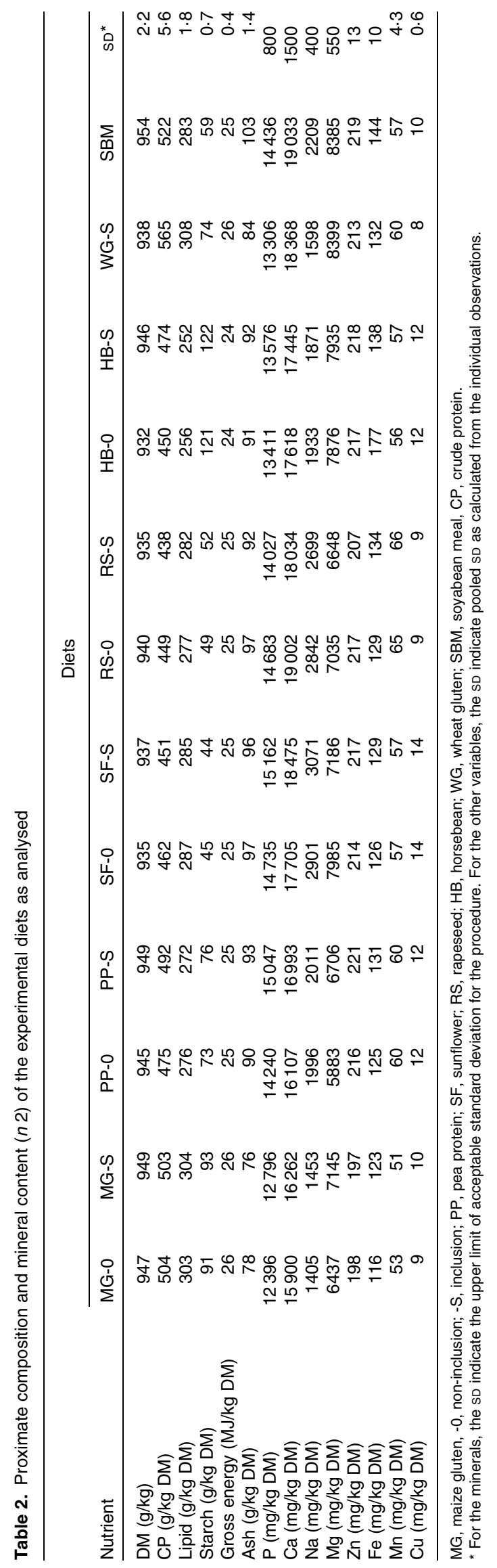


FER during the present experimental period. OSI were calculated as percentages of the weight of the organ in relation to body weight. AD was estimated by the indirect method using $\mathrm{Y}_{2} \mathrm{O}_{3}$ as an inert marker ${ }^{(22)}$ and calculated as: $\mathrm{AD}_{\mathrm{N}}=$ $100-\left(100 \times\left(M_{\text {feed }} / M_{\text {faeces }}\right) \times\left(N_{\text {faeces }} / N_{\text {feed }}\right)\right)$, where $M_{\text {feed }}$ and $M_{\text {faeces }}$ are percentage concentration of the inert marker $\left(\mathrm{Y}_{2} \mathrm{O}_{3}\right)$ in feed and faeces, respectively, and $N_{\text {feed }}$ and $N_{\text {faeces }}$ represent percentage concentration of a nutrient in feed and faeces, respectively. Nutrient retention - retentions of $\mathrm{CP}$, individual amino acids and energy - was calculated as: $100 \times\left(\left(\mathrm{FBW} \times C_{1}\right)-\left(\mathrm{IBW} \times C_{0}\right)\right) \times\left(F \times C_{\text {diet }}\right)^{-1}$, where $C_{\text {diet }}$ is the content in the diets, and $C_{0}$ and $C_{1}$ are the initial and final contents in the fish.

\section{Statistical analyses}

The results were analysed using SAS Enterprise Guide 4.1 statistical software (SAS Institute, Inc., Cary, NC, USA). Tank means were used as the statistical unit in the analyses because individual fish responses were not considered independent within a tank. Saponin inclusion and basal diet were evaluated as class variables in a two-way ANOVA with interaction. Significant interaction between the main effects was observed for many variables and a one-way ANOVA was then used as an aid for the interpretation of data. When the interaction was significant, effects of saponin supplementation were evaluated based on differences in effect within the various basal diets, whereas effects of basal diet were evaluated based on the differences observed between fish fed the unsupplemented diets, i.e. MG-0, pea protein (PP-0), sunflower (SF-0), rapeseed (RS-0) and horsebean (HB-0). When there was no significant interaction, the basal diets were compared according to their main effects and mentioned as MG, PPC, SFM, RSM and HBM. The one-way ANOVA also allowed inclusion in the comparison of the SBM and the
WG-S diets that were excluded from the two-way ANOVA. The level of significance was set at $P<0.05$, and $P$-values between 0.05 and $0 \cdot 1$ were considered as indications of effects mentioned as trends. The Duncan's multiple range test was employed as the mean separating technique.

\section{Results}

The results for the two observation periods were generally similar but the effects were less clear after the first period, day $0-30$. Results that follow are presented with main emphasis on the observations for the period, day 31-80. In the following presentation of results, under each subheading, the effects of saponin supplementation are presented first; thereafter, considerations regarding the basal diets are given. The basal diets are presented and discussed using abbreviations; MG represents the basal diet with MG and is used when there was no significant interaction between saponin supplementation and basal diet. When the interaction was significant, the results for the unsupplemented diet is given and presented as MG-0. Similar abbreviations are used for the other basal diets. When the protein source is mentioned as such, the full term is used.

\section{Reference diets}

The fish fed the SBM reference diets showed all the expected signs of enteritis usually observed in the DI of Atlantic salmon and confirmed that the experiment had the right conditions to reveal if saponins are involved in the development of this condition. The signs were: reduced bile salt concentration, low activity of BBM LAP, high chyme trypsin activity, reduced OSI, reduced mucosal fold height, increased width and cell infiltration of lamina propria and submucosa, etc. ${ }^{(1,19)}$. The results for the other reference diet, the WG-S, did not add

Table 3. Amino acid composition $(n 2)$ in the experimental diets

\begin{tabular}{|c|c|c|c|c|c|c|c|c|c|c|c|c|c|}
\hline & \multicolumn{13}{|c|}{ Diets (\% of protein) } \\
\hline & MG-0 & MG-S & PP-0 & PP-S & SF-0 & SF-S & RS-0 & RS-S & HB-O & HB-S & WG-S & SBM & Pooled SD \\
\hline \multicolumn{14}{|c|}{ Essential amino acids } \\
\hline Arg & 5.4 & $5 \cdot 5$ & $7 \cdot 7$ & $7 \cdot 8$ & $7 \cdot 3$ & $7 \cdot 3$ & $6 \cdot 8$ & $6 \cdot 8$ & $7 \cdot 6$ & $7 \cdot 6$ & $5 \cdot 7$ & $7 \cdot 2$ & 0.08 \\
\hline $\mathrm{His}$ & $2 \cdot 7$ & $2 \cdot 7$ & $3 \cdot 0$ & 3.0 & $3 \cdot 1$ & $3 \cdot 1$ & $3 \cdot 1$ & $3 \cdot 1$ & $3 \cdot 1$ & $3 \cdot 1$ & $2 \cdot 8$ & $3 \cdot 1$ & 0.04 \\
\hline Ile & 4.3 & 4.3 & 4.5 & 4.4 & 4.6 & 4.5 & 4.6 & 4.5 & 4.6 & 4.5 & $4 \cdot 3$ & $4 \cdot 7$ & 0.06 \\
\hline Leu & $10 \cdot 9$ & $10 \cdot 9$ & 8.0 & 8.0 & 7.9 & 7.9 & 8.0 & 8.0 & $8 \cdot 1$ & $8 \cdot 1$ & $7 \cdot 8$ & $8 \cdot 1$ & $0 \cdot 16$ \\
\hline Lys & $6 \cdot 2$ & $6 \cdot 3$ & $8 \cdot 1$ & $8 \cdot 3$ & $7 \cdot 8$ & $7 \cdot 8$ & 8.0 & $8 \cdot 0$ & $8 \cdot 2$ & $8 \cdot 2$ & $6 \cdot 3$ & $8 \cdot 1$ & 0.09 \\
\hline Met & $2 \cdot 7$ & $2 \cdot 8$ & $3 \cdot 3$ & $3 \cdot 2$ & $3 \cdot 1$ & $3 \cdot 1$ & 3.0 & 3.0 & $2 \cdot \overline{7}$ & $2 \cdot 7$ & 2.6 & 2.9 & 0.05 \\
\hline Phe & $5 \cdot 1$ & $5 \cdot 3$ & $4 \cdot 8$ & $5 \cdot 0$ & $4 \cdot 7$ & 4.9 & 4.6 & $4 \cdot 8$ & 4.6 & $4 \cdot 8$ & $5 \cdot 0$ & 4.9 & $0 \cdot 10$ \\
\hline Thr & 3.9 & 3.9 & 4.2 & 4.2 & 4.4 & 4.4 & 4.5 & 4.5 & 4.3 & 4.2 & 3.7 & 4.3 & 0.06 \\
\hline Trp & 1.0 & 1.0 & $1 \cdot 2$ & $1 \cdot 2$ & 1.3 & 1.3 & 1.4 & 1.3 & 1.3 & $1 \cdot 2$ & $1 \cdot 2$ & 1.3 & 0.03 \\
\hline Val & $5 \cdot 1$ & $5 \cdot 1$ & $5 \cdot 3$ & $5 \cdot 2$ & 5.5 & 5.4 & $5 \cdot 6$ & 5.5 & 5.4 & $5 \cdot 3$ & 4.9 & 5.4 & 0.08 \\
\hline \multicolumn{14}{|c|}{ Non-essential amino acids } \\
\hline Ala & $6 \cdot 6$ & $6 \cdot 6$ & 5.5 & 5.4 & $5 \cdot 8$ & $5 \cdot 8$ & $5 \cdot 8$ & $5 \cdot 8$ & $5 \cdot 7$ & 5.7 & $4 \cdot 8$ & 5.7 & $0 \cdot 10$ \\
\hline$A s p+A s n$ & $8 \cdot 3$ & $8 \cdot 3$ & 10.4 & 10.5 & 9.9 & 9.9 & 9.6 & $9 \cdot 6$ & $10 \cdot 3$ & 10.2 & 7.5 & $10 \cdot 4$ & 0.12 \\
\hline Cys & $1 \cdot 2$ & $1 \cdot 2$ & 1.0 & 1.0 & 1.0 & 1.0 & $1 \cdot 2$ & $1 \cdot 2$ & 1.0 & 1.0 & $1 \cdot 3$ & 1.0 & 0.02 \\
\hline Glu + Gln & $17 \cdot 3$ & $17 \cdot 1$ & $15 \cdot 5$ & $15 \cdot 4$ & $15 \cdot 7$ & $15 \cdot 6$ & $15 \cdot 3$ & $15 \cdot 3$ & $15 \cdot 2$ & $15 \cdot 5$ & $22 \cdot 8$ & $15 \cdot 5$ & 0.26 \\
\hline Gly & $4 \cdot 6$ & 4.6 & $5 \cdot 1$ & $5 \cdot 2$ & 5.9 & 5.9 & $5 \cdot 8$ & $5 \cdot 8$ & $5 \cdot 5$ & $5 \cdot 5$ & 4.9 & $5 \cdot 5$ & 0.07 \\
\hline Pro & $6 \cdot 2$ & $6 \cdot 1$ & 4.4 & $4 \cdot 2$ & 4.6 & 4.5 & $5 \cdot 0$ & 4.9 & 4.6 & 4.4 & $6 \cdot 8$ & 4.4 & $0 \cdot 17$ \\
\hline Ser & 4.4 & 4.4 & 4.4 & 4.5 & 4.3 & 4.3 & 4.3 & $4 \cdot 3$ & 4.4 & 4.4 & 4.3 & $4 \cdot 3$ & 0.08 \\
\hline Tyr & 4.0 & 4.0 & $3 \cdot 6$ & 3.5 & 3.3 & 3.2 & 3.3 & $3 \cdot 3$ & 3.4 & 3.4 & 3.3 & 3.4 & 0.08 \\
\hline
\end{tabular}

MG, maize gluten, -0, non-inclusion; -S, inclusion; PP, pea protein; SF, sunflower; RS, rapeseed; HB, horsebean; WG, wheat gluten; SBM, soyabean meal. 
important information to the results obtained with the basal diets. The results for the SBM and WG-S are therefore not further presented here, except in a few cases.

Feed intake. Supplementation of the basal diets with saponins affected DM feed intake, but the effect was dependent on the basal diet (Table 4). The saponin-basal diet interaction was significant in the second feeding period, day 31-80. Inclusion of saponins significantly decreased feed intake for the PPC basal diet, but for the other basal diets feed intake seemed to be constant or to slightly increase, especially in the case of SFM basal diet for which the increase was significant.

Fish fed different basal diets differed in feed intake. In the first period, feed intake was highest in fish fed SF-0 and PP-0, and lowest in fish fed HB-0. In the second feeding period, there was a general increase in feed intake for all basal diets, with the HB-0 showing the highest increase of about $100 \%$. The highest feed intakes were observed for fish fed PP-0 and HB-0. The fish fed RS-0 had the lowest intake.

Body weight and thermal-unit growth coefficient. As for feed intake, there was a significant interaction between saponin supplementation and basal diet for body weight and TGC (see Table 4). Fish fed the PP-S diet showed significantly lower values than fish fed the PP-0 diet both at day 30 and at the final sampling. For the other basal diets, saponin supplementation rather increased growth, but not significantly.

There were significant differences between the basal diets regarding growth parameters observed on day 30. Fish fed diet PP-0 had the highest values, while fish fed RS-0 had the lowest. Also at termination, fish fed PP-0 had the highest weight and TGC, and fish fed RS-0 remained the smallest. The ranking of the basal diets for TGC was as follows: PP-O $0^{\mathrm{a}}>\mathrm{HB}-0^{\mathrm{a}, \mathrm{b}}>\mathrm{MG}-0^{\mathrm{a}, \mathrm{b}, \mathrm{c}, \mathrm{d}}>\mathrm{SF}-0^{\mathrm{c}, \mathrm{d}, \mathrm{e}}>\mathrm{RS}-0^{\mathrm{e}}$ (diets with different superscripts differed significantly). The HB-O and SF-0 had switched place compared to the first period, and were now significantly different.

Feed efficiency ratio. Saponin supplementation did not significantly affect the FER (see Table 4) for any of the basal diets. Differences in FER were caused by differences between the basal diets. During the first $30 \mathrm{~d}$ of the trial, FER was highest for fish fed PPC followed by MG and HBM with no significant difference between the three. The RSM had the lowest FER, significantly lower than the other basal diets. In the second feeding period, the picture was somewhat different with the MG showing the highest FER followed by PPC, HBM, SFM and RSM. The latter two differed significantly from the MG.

\section{Apparent nutrient digestibility}

Macronutrients. Saponin inclusion was associated with a small but significant reduction in AD of lipids (Table 5). The effect was most pronounced for the PPC, but the overall saponin-basal diet interaction was not significant. Saponin supplementation also affected protein $\mathrm{AD}$, but the effect depended on the basal diet. For the PPC diet, the effect was negative. A negative trend was also apparent for RSM. For MG and HBM, the trend was positive, but only marginally. Saponin inclusion did not affect starch AD significantly. For energy $\mathrm{AD}$, the saponin effect was clearly negative for the PPC and RSM, whereas for the other basal diets, the trend was positive. Regarding faecal DM (FDM), significant negative effects of saponin supplementation were seen, depending on the basal diet. The effect was greatest for PPC, for which saponin supplementation reduced FDM by $6 \%$ units. For SFM, RSM and HBM, the reduction was about $1 \%$ unit, whereas for $M G$, the reduction was negligible.

Significant differences were observed regarding AD of all the macronutrients and energy due to differences between the basal diets. Fish fed MG-0 had the highest protein and energy AD; fish fed RS-0, the lowest. For lipid AD, the highest value was observed in fish fed PPC diets, being significantly higher than all the other basal diets, whereas the lowest value was observed in fish fed SFM diets. The largest variation in nutrient $\mathrm{AD}$ was observed for starch. The highest value was observed with MG and the lowest with HBM. FDM was about $14 \%$ for most basal diets, except the HBM (observed for the HB-0), which showed significantly higher FDM than the other supplemented diets.

\section{Amino acids}

Essential amino acids. Generally, AD coefficients for all of the essential amino acids (EAA) were high, predominantly above $90 \%$ (see Table 6). Saponin inclusion effects on individual EAA differed and depended on the basal diet. For MG, saponin inclusion was consistently associated with a significantly higher AD for all EAA except Leu. Even for Leu, the trend was maintained as the MG-S diet had a numerically higher AD than the MG-0 diet. Saponin inclusion for the rest of the basal diets had either a negative effect on $\mathrm{AD}$ of the EAA or no effect. For PPC, the AD of all EAA was markedly lower for fish fed the saponin-supplemented diet. The pattern was similar for the RSM, except that differences in the AD of His, Lys and Phe were not significant. There were no clear saponin supplementation effects for SFM and HBM for any of the EAA.

The MG-0 and HB-0 generally showed high $\mathrm{AD}$ values across all the EAA, and $\mathrm{AD}$ of Leu, Trp and Phe were significantly higher for MG-0 than for HB-0. Also, the PP-0 diet showed quite high amino acid $\mathrm{AD}$. In contrast, fish fed the RS-0 consistently had the lowest AD values for all the EAA, while fish fed the SF-0 were intermediate. Among the EAA, Trp showed the lowest values.

Non-essential amino acids and taurine. Generally, AD of the non-essential amino acids (NEAA) were not as high as for the EAA (see Table 7). Saponin effects were similar to those observed for the EAA AD values. Saponin inclusion in the MG basal diet was associated with significantly higher digestibilities of Ala, Asp-Asn and Gly, and with numerically higher values for the remaining NEAA. Saponin inclusion in the PPC showed significantly lower AD in the saponin-supplemented diet for all the NEAA. The greatest difference was seen for Cys for which AD dropped 12\% units due to the saponin inclusion. Saponin inclusion was also associated with significantly lower AD for RSM diets for most NEAA except Cys and Asp-Asn.

The AD of all NEAA were generally highest for fish fed the MG-0 diet and lowest for fish fed the RS-0 diet. The lowest AD and the greatest differences were found for Cys. 
Table 4. Feed intake, growth and feed utilisation efficiency of Atlantic salmon during the feeding period

\begin{tabular}{|c|c|c|c|c|c|c|c|c|c|}
\hline & \multicolumn{3}{|c|}{ BW (g) } & \multicolumn{2}{|c|}{ DM intake (\% mean BW/d) } & \multicolumn{2}{|c|}{$\mathrm{TGC} \times 1000$} & \multicolumn{2}{|c|}{ FER (DM basis, g/g) } \\
\hline & Day 0 & Day 30 & Day 80 & Day 0-30 & Day 31-80 & Day $0-30$ & Day $31-80$ & Day 0-30 & Day $31-80$ \\
\hline \multicolumn{10}{|c|}{ Two-way ANOVA model } \\
\hline$P$ (model) & $0 \cdot 18$ & $0 \cdot 16$ & 0.01 & 0.02 & 0.002 & $0 \cdot 10$ & 0.0004 & 0.05 & 0.01 \\
\hline Pooled SEM & 1.4 & 9.5 & $25 \cdot 8$ & 0.04 & 0.03 & 0.2 & $0 \cdot 1$ & 0.8 & 0.04 \\
\hline \multicolumn{10}{|c|}{$P$ values effect tests in the two-way ANOVA model } \\
\hline Saponin & 0.99 & 0.87 & 0.73 & 0.36 & 0.19 & 0.89 & 0.25 & 0.55 & 0.64 \\
\hline Basal diet & 0.08 & $0 \cdot 12$ & 0.01 & 0.01 & 0.002 & 0.06 & 0.0001 & 0.01 & 0.001 \\
\hline Interaction & 0.34 & $0 \cdot 18$ & 0.03 & 0.09 & 0.01 & $0 \cdot 16$ & 0.01 & 0.37 & 0.27 \\
\hline \multicolumn{10}{|c|}{ Marginal means for the two-way ANOVA model } \\
\hline \multicolumn{10}{|c|}{ Saponin supplementation } \\
\hline No saponin & 262 & 319 & 537 & 0.52 & 0.78 & 1.09 & 2.63 & $1 \cdot 18$ & 1.34 \\
\hline With saponin & 262 & 318 & 543 & 0.54 & 0.80 & 1.07 & $2 \cdot 72$ & $1 \cdot 14$ & 1.35 \\
\hline \multicolumn{10}{|l|}{ Basal diet } \\
\hline MG & 262 & 318 & $547^{\mathrm{a}}$ & $0.50^{b, c}$ & $0.77^{b, c}$ & 1.09 & $2 \cdot 79^{\mathrm{a}, \mathrm{b}}$ & $1 \cdot 24^{\mathrm{a}}$ & $1.45^{\mathrm{a}}$ \\
\hline PPC & 260 & 330 & $578^{\mathrm{a}}$ & $0.58^{a, b}$ & $0 \cdot 81^{b}$ & 1.34 & $2 \cdot 87^{\mathrm{a}, \mathrm{b}}$ & $1 \cdot 28^{\mathrm{a}}$ & $1 \cdot 39^{a, b}$ \\
\hline SFM & 263 & 325 & $549^{\mathrm{a}}$ & $0.60^{\mathrm{a}}$ & $0.81^{\mathrm{b}}$ & 1.20 & $2 \cdot 64^{b}$ & $1 \cdot 14^{\mathrm{a}}$ & $1 \cdot 31^{b, c}$ \\
\hline RSM & 262 & 303 & $461^{\mathrm{b}}$ & $0.52^{\mathrm{a}, \mathrm{b}, \mathrm{c}}$ & $0.71^{\mathrm{c}}$ & 0.81 & $2 \cdot 06^{\mathrm{C}}$ & $0.89^{\mathrm{b}}$ & $1 \cdot 22^{\mathrm{c}}$ \\
\hline HBM & 265 & 314 & $563^{\mathrm{a}}$ & $0.44^{\mathrm{c}}$ & $0.87^{\mathrm{a}}$ & 0.95 & $3.01^{a}$ & $1 \cdot 23^{\mathrm{a}}$ & $1.37^{\mathrm{a}, \mathrm{b}}$ \\
\hline \multicolumn{10}{|c|}{ Means of the diets for the one-way ANOVA model } \\
\hline MG-0 & 261 & $316^{\mathrm{c}}$ & $529^{b, c, d}$ & $0.49^{\mathrm{c}, \mathrm{d}, \mathrm{e}}$ & $0.76^{\mathrm{b}, \mathrm{c}, \mathrm{d}}$ & $1.07^{b, c}$ & $2 \cdot 67^{\mathrm{a}, \mathrm{b}, \mathrm{c}, \mathrm{d}}$ & $1.25^{\mathrm{a}}$ & $1.42^{\mathrm{a}, \mathrm{b}, \mathrm{c}}$ \\
\hline MG-S & 264 & $321^{a, b, c}$ & $566^{a, b, c}$ & $0.52^{\mathrm{c}, \mathrm{d}, \mathrm{e}}$ & $0.78^{b, c, d}$ & $1 \cdot 11^{b, c}$ & $2 \cdot 92^{\mathrm{a}, \mathrm{b}, \mathrm{c}}$ & $1.22^{\mathrm{a}}$ & $1.48^{\mathrm{a}, \mathrm{b}}$ \\
\hline PP-0 & 260 & $347^{\mathrm{a}, \mathrm{b}}$ & $640^{\mathrm{a}}$ & $0.65^{a, b}$ & $0.86^{a, b}$ & $1 \cdot 63^{\mathrm{a}}$ & $3 \cdot 19^{\mathrm{a}}$ & $1.42^{\mathrm{a}}$ & $1.41^{a, b, c, d}$ \\
\hline PP-S & 260 & $313^{\mathrm{C}}$ & $517^{b, c, d}$ & $0.52^{\mathrm{c}, \mathrm{d}, \mathrm{e}}$ & $0.75^{c, d}$ & $1.04^{b, c}$ & $2 \cdot 56^{\mathrm{b}, \mathrm{c}, \mathrm{d}}$ & $1 \cdot 15^{\mathrm{a}, \mathrm{b}}$ & $1 \cdot 38^{\mathrm{b}, \mathrm{c}, \mathrm{d}}$ \\
\hline SF-0 & 264 & $320^{a, b, c}$ & $518^{\mathrm{b}, \mathrm{c}, \mathrm{d}}$ & $0.55^{\mathrm{b}, \mathrm{c}, \mathrm{d}}$ & $0.72^{d}$ & $1.09^{b, c}$ & $2 \cdot 40^{\mathrm{c}, \mathrm{d}, \mathrm{e}}$ & $1 \cdot 13^{\mathrm{a}, \mathrm{b}}$ & $1 \cdot 34^{\mathrm{c}, \mathrm{d}}$ \\
\hline SF-S & 262 & $330^{a, b, c}$ & $580^{\mathrm{a}, \mathrm{b}}$ & $0.65^{\mathrm{a}, \mathrm{b}}$ & $0.89^{\mathrm{a}}$ & $1.31^{a, b}$ & $2 \cdot 89^{\mathrm{a}, \mathrm{b}, \mathrm{c}}$ & $1 \cdot 15^{\mathrm{a}, \mathrm{b}}$ & $1.27^{\mathrm{d}, \mathrm{e}}$ \\
\hline RS-0 & 263 & $302^{c}$ & $448^{d}$ & $0.48^{\mathrm{c}, \mathrm{d}, \mathrm{e}}$ & $0.71^{d}$ & $0 \cdot 76^{c}$ & $1.95^{\mathrm{e}}$ & $0.91^{b}$ & $1 \cdot 16^{\mathrm{e}}$ \\
\hline RS-S & 261 & $305^{c}$ & $475^{c, d}$ & $0.56^{\mathrm{b}, \mathrm{c}}$ & $0.71^{d}$ & $0 \cdot 86^{b, c}$ & $2 \cdot 18^{\mathrm{d}, \mathrm{e}}$ & $0.87^{b}$ & $1 \cdot 28^{\mathrm{d}, \mathrm{e}}$ \\
\hline HB-0 & 264 & $309^{c}$ & $551^{a, b, c}$ & $0.42^{\mathrm{e}}$ & $0.85^{a, b, c}$ & $0.87^{\mathrm{b}, \mathrm{c}}$ & $2 \cdot 95^{a, b}$ & $1 \cdot 17^{\mathrm{a}, \mathrm{b}}$ & $1 \cdot 38^{\mathrm{b}, \mathrm{c}, \mathrm{d}}$ \\
\hline HB-S & 265 & $319^{b, c}$ & $576^{a, b}$ & $0.46^{\mathrm{c}, \mathrm{d}, \mathrm{e}}$ & $0.89^{\mathrm{a}}$ & $1.04^{b, c}$ & $3 \cdot 07^{\mathrm{a}, \mathrm{b}}$ & $1 \cdot 30^{\mathrm{a}}$ & $1 \cdot 36^{\mathrm{b}, \mathrm{c}, \mathrm{d}}$ \\
\hline WG-S & 264 & $319^{b, c}$ & $568^{a, b, c}$ & $0.44^{\mathrm{d}, \mathrm{e}}$ & $0.75^{\mathrm{c}, \mathrm{d}}$ & $1.05^{\mathrm{b}, \mathrm{c}}$ & $2 \cdot 91^{a, b, c}$ & $1.38^{\mathrm{a}}$ & $1.52^{\mathrm{a}}$ \\
\hline SBM & 264 & $350^{\mathrm{a}}$ & $605^{\mathrm{a}, \mathrm{b}}$ & $0.72^{\mathrm{a}}$ & $0.79^{\mathrm{a}, \mathrm{b}, \mathrm{c}, \mathrm{d}}$ & $1.61^{\mathrm{a}}$ & $2 \cdot 82^{\mathrm{a}, \mathrm{b}, \mathrm{c}}$ & $1 \cdot 26^{\mathrm{a}}$ & $1 \cdot 37^{\mathrm{b}, \mathrm{c}, \mathrm{d}}$ \\
\hline
\end{tabular}

BW, body weight; TGC, thermal growth coefficient, FER, feed efficiency ratio; MG, maize gluten; PPC, pea protein concentrate; SFM, sunflower meal; RSM, rapeseed meal; HBM, horsebean meal; -0, non-inclusion; -S, inclusion; PP, pea protein; SF, sunflower; RS, rapeseed; HB, horsebean; WG, wheat gluten; SBM, soyabean meal.

a,b,c,d,e Mean values within a column with unlike superscript letters were significantly different $(P<0.05)$. 
The AD of taurine, a sulfonic acid found in high concentration in both pancreatic juice and bile, is worth mentioning. There was no significant effect of saponin supplementation, but the PPC and RSM diets showed significantly lower taurine $\mathrm{AD}$ than the other basal diets.

Fatty acids. The AD of fatty acids are presented in Table 8. Saponin supplementation decreased AD of most fatty acids, significantly for $16: 1 n-7,18: 0,18: 1 n-9,20: 2 n-6,22: 1 n-11$ and sum MUFA. The same trend was seen for all other fatty acids except $18: 1 n-11$ and $18: 3 n-3$. No significant interactions between saponins and basal diet were observed.

The $\mathrm{AD}$ of most of the fatty acids were influenced by the basal diet. The trends for the SFA such as 16:0 and 18:0 were similar. The HBM and PPC showed the highest values, RSM and MG the lowest, significantly lower than the former two. The SFM showed intermediate values and did not differ from the other basal diets. The AD of MUFA was generally high, with PPC showing the highest in this category. The SFM generally showed the lowest $\mathrm{AD}$, and the difference between the two was significant for most of the MUFA. In between, MG, RSM and HBM mostly ranked 2, 3 and 4 without being significantly different, but for some of the MUFA significant differences compared to PPC and SFM were observed. The AD of the $n-6$ and $n-3$ generally showed the same ranking of the basal diets as the MUFA.
Minerals. The AD values for minerals are presented in Table 9. They represent the result of intake of minerals from seawater and diet in addition to mineral absorption and secretion by the intestine. Sea water may supply the fish with more $\mathrm{Na}$, $\mathrm{Ca}$ and $\mathrm{Mg}$ than the diet and consequently $\mathrm{AD}$ for these minerals may become negative, which was the case in particular for $\mathrm{Mg}$. Also, AD for Mn showed several negative values, and for $\mathrm{AD}$ of the sum of all minerals, i.e. ash, many values were negative.

Saponin inclusion negatively affected the AD of $\mathrm{Na}$ and $\mathrm{Mg}$ for PPC, RSM and SFM. Ash AD showed the same picture and a similar trend was apparent for $\mathrm{AD}$ of $\mathrm{Ca}$. For MG and HBM, there was no clear effect. Saponin supplementation increased or tended to increase $\mathrm{AD}$ of $\mathrm{Cu}, \mathrm{Fe}, \mathrm{Mn}, \mathrm{Zn}$ and $\mathrm{P}$ for MG and SFM, and decreased AD of these minerals for RSM. For PPC and HBM, the results varied between the minerals. The only significant effects for these two basal diets were a positive effect of saponins for PPC on $\mathrm{AD}$ of $\mathrm{Cu}$ and a negative effect for HBM on Fe.

Significant differences were observed between the basal diets for all minerals except $\mathrm{Ca}$. For $\mathrm{Na}$, fish fed the HB-0 and MG-0 diets had the highest $\mathrm{AD}$, significantly different from fish fed PP-0 and RS-0 diets which had the lowest. Fish fed the SF-0 diet showed intermediate values, not significantly different from other fish. Fish fed HB-0 also showed the highest $\mathrm{AD}$ of $\mathrm{Mg}$, but the value was significantly different only

Table 5. Apparent digestibility of macronutrients and energy, and faecal DM (FDM) for Atlantic salmon fed the experimental diets (\%)

\begin{tabular}{|c|c|c|c|c|c|}
\hline & Crude protein & Lipid & Starch & Energy & FDM \\
\hline \multicolumn{6}{|c|}{ Two-way ANOVA model } \\
\hline$P($ model $)$ & $<0.0001$ & 0.0487 & $<0.0001$ & $<0.0001$ & $<0.0001$ \\
\hline Pooled SEM & 0.5 & 0.4 & 1.6 & 0.5 & 0.4 \\
\hline \multicolumn{6}{|c|}{$P$ values effect tests in the two-way ANOVA model } \\
\hline Saponin & 0.46 & 0.05 & 0.58 & 0.15 & $<0.0001$ \\
\hline Basal diet & $<0.0001$ & 0.04 & $<0.0001$ & $<0.0001$ & $<0.0001$ \\
\hline Interaction & 0.05 & 0.20 & 0.10 & 0.02 & $<0.0001$ \\
\hline \multicolumn{6}{|c|}{ Marginal means for the two-way ANOVA model } \\
\hline \multicolumn{6}{|c|}{ Saponin supplementation } \\
\hline No saponin & $86 \cdot 1$ & $96 \cdot 1^{\mathrm{a}}$ & 81.5 & $84 \cdot 3$ & $14.9^{\mathrm{a}}$ \\
\hline With saponin & 85.9 & $95 \cdot 6^{\mathrm{b}}$ & $80 \cdot 9$ & 83.8 & $13 \cdot 1^{\mathrm{b}}$ \\
\hline \multicolumn{6}{|l|}{ Basal diet } \\
\hline MG & $89 \cdot 2^{\mathrm{a}}$ & $95 \cdot 7^{\mathrm{b}}$ & $82 \cdot 7^{\mathrm{b}}$ & $89 \cdot 5^{\mathrm{a}}$ & $13 \cdot 9^{\mathrm{b}}$ \\
\hline PPC & $86 \cdot 3^{\mathrm{b}}$ & $96 \cdot 6^{\mathrm{a}}$ & $84 \cdot 2^{\mathrm{b}}$ & $85 \cdot 1^{\mathrm{b}}$ & $11 \cdot 1^{\mathrm{c}}$ \\
\hline SFM & $85 \cdot 9^{\mathrm{b}}$ & $95 \cdot 3^{\mathrm{b}}$ & $81 \cdot 8^{\mathrm{b}}$ & $82 \cdot 4^{\mathrm{C}}$ & $14 \cdot 1^{\mathrm{b}}$ \\
\hline RSM & $82 \cdot 0^{\mathrm{C}}$ & $95 \cdot 6^{\mathrm{b}}$ & $89 \cdot 7^{a}$ & $79 \cdot 8^{d}$ & $13 \cdot 9^{b}$ \\
\hline HBM & $86 \cdot 6^{\mathrm{b}}$ & $96 \cdot 2^{\mathrm{ab}}$ & $67 \cdot 7^{\mathrm{C}}$ & $83 \cdot 5^{\mathrm{c}}$ & $16 \cdot 9^{a}$ \\
\hline \multicolumn{6}{|c|}{ Means of the diets for the one-way ANOVA model } \\
\hline MG-0 & $88 \cdot 6^{\mathrm{b}, \mathrm{c}}$ & $95 \cdot 5^{\mathrm{b}}$ & $80 \cdot 3^{\mathrm{d}, \mathrm{e}}$ & $89 \cdot 0^{\mathrm{b}}$ & $13 \cdot 9^{b, c, d}$ \\
\hline MG-S & $89.9^{b}$ & $95 \cdot 9^{\mathrm{b}}$ & $85 \cdot 2^{\mathrm{b}, \mathrm{c}, \mathrm{d}}$ & $90 \cdot 1^{\mathrm{b}}$ & $13 \cdot 8^{\mathrm{c}, \mathrm{d}}$ \\
\hline PP-0 & $87 \cdot 4^{c, d}$ & $97 \cdot 3^{a}$ & $83 \cdot 8^{\mathrm{c}, \mathrm{d}}$ & $86 \cdot 2^{c}$ & $14 \cdot 1^{\mathrm{b}, \mathrm{c}, \mathrm{d}}$ \\
\hline PP-S & $85 \cdot 1^{\mathrm{e}}$ & $95 \cdot 9^{\mathrm{b}}$ & $84 \cdot 6^{\mathrm{b}, \mathrm{c}, \mathrm{d}}$ & $84 \cdot 1^{d}$ & $8 \cdot 1^{\dagger}$ \\
\hline SF-0 & $85 \cdot 9^{\mathrm{d}, \mathrm{e}}$ & $95 \cdot 6^{\mathrm{b}}$ & $82.9^{d}$ & $82 \cdot 2^{e, f}$ & $14 \cdot 6^{\mathrm{b}, \mathrm{c}}$ \\
\hline SF-S & $85 \cdot 9^{\mathrm{d}, \mathrm{e}}$ & $95 \cdot 0^{\mathrm{b}}$ & $80 \cdot 8^{\mathrm{d}, \mathrm{e}}$ & $82 \cdot 6^{\mathrm{d}, \mathrm{e}}$ & $13 \cdot 5^{\mathrm{c}, \mathrm{d}}$ \\
\hline RS-0 & $82 \cdot 6^{f}$ & $96 \cdot 1^{\mathrm{b}}$ & $90 \cdot 4^{a}$ & $80 \cdot 8^{f}$ & $14 \cdot 4^{\mathrm{b}, \mathrm{c}, \mathrm{d}}$ \\
\hline RS-S & $81.5^{\dagger}$ & $95 \cdot 2^{\mathrm{b}}$ & $89 \cdot 0^{a, b}$ & $78 \cdot 7^{9}$ & $13 \cdot 5^{\mathrm{d}}$ \\
\hline HB-O & $86 \cdot 3^{\mathrm{d}, \mathrm{e}}$ & $96 \cdot 2^{b}$ & $70 \cdot 3^{f}$ & $83 \cdot 4^{d, e}$ & $17 \cdot 3^{a}$ \\
\hline HB-S & $87.0^{\mathrm{d}}$ & $96 \cdot 1^{\mathrm{b}}$ & $65 \cdot 2^{\mathrm{g}}$ & $83 \cdot 6^{\mathrm{d}, \mathrm{e}}$ & $16 \cdot 5^{a}$ \\
\hline WG-S & $92 \cdot 4^{\mathrm{a}}$ & $95 \cdot 6^{\mathrm{b}}$ & $77 \cdot 3^{\mathrm{e}}$ & $91 \cdot 7^{\mathrm{a}}$ & $15 \cdot 0^{\mathrm{b}}$ \\
\hline SBM & $86 \cdot 9^{d}$ & $95 \cdot 3^{\mathrm{b}}$ & $88 \cdot 4^{a, b, c}$ & $86 \cdot 3^{\mathrm{C}}$ & $9.5^{\mathrm{e}}$ \\
\hline
\end{tabular}

MG, maize gluten; PPC, pea protein concentrate; SFM, sunflower meal; RSM, rapeseed meal; HBM, horsebean meal; -0, non-inclusion; -S, inclusion; PP, pea protein; SF, sunflower; RS, rapeseed; HB, horsebean; WG, wheat gluten; SBM, soyabean meal.

$a, b, c, d, e, f, g$ Mean values within a column with unlike superscript letters were significantly different $(P<0.05)$. 
Table 6. Apparent digestibility of essential amino acids for Atlantic salmon fed the experimental diets (\%)

\begin{tabular}{|c|c|c|c|c|c|c|c|c|c|c|}
\hline & Lys & Met & $\operatorname{Arg}$ & Leu & Trp & His & lle & Phe & Thr & Val \\
\hline \multicolumn{11}{|c|}{ Two-way ANOVA model } \\
\hline$P$ (model) & $<0.0001$ & $<0.0001$ & $<0.0001$ & $<0.0001$ & $<0.0001$ & $<0.0001$ & 0.001 & $<0.0001$ & $<0.0001$ & $<0.0001$ \\
\hline Pooled SEM & 0.4 & 0.3 & 1.0 & 0.4 & 0.4 & 0.6 & 0.3 & 0.5 & 0.3 & 0.3 \\
\hline \multicolumn{11}{|c|}{$P$ values effect tests in the two-way ANOVA model } \\
\hline Saponin & 0.01 & 0.01 & 0.01 & 0.004 & 0.04 & 0.26 & 0.29 & 0.03 & 0.14 & 0.01 \\
\hline Basal diet & $<0.0001$ & $<0.0001$ & $<0.0001$ & $<0.0001$ & $<0.0001$ & $<0.0001$ & 0.0002 & $<0.0001$ & $<0.0001$ & $<0.0001$ \\
\hline Interaction & 0.001 & 0.002 & 0.0002 & 0.0004 & 0.004 & 0.01 & 0.01 & 0.002 & 0.002 & 0.001 \\
\hline \multicolumn{11}{|c|}{ Marginal means for the two-way ANOVA model } \\
\hline \multicolumn{11}{|c|}{ Saponin supplementation } \\
\hline No saponin & $92.5^{\mathrm{a}}$ & $90 \cdot 8^{\mathrm{a}}$ & $92 \cdot 8^{\mathrm{a}}$ & $92.0^{\mathrm{a}}$ & $86 \cdot 3^{\mathrm{a}}$ & $90 \cdot 3$ & 90.4 & $90 \cdot 0$ & $88 \cdot 2^{\mathrm{a}}$ & $90 \cdot 5^{\mathrm{a}}$ \\
\hline With saponin & $92 \cdot 3^{\mathrm{b}}$ & $90 \cdot 6^{\mathrm{b}}$ & $92 \cdot 6^{\mathrm{b}}$ & $91.5^{\mathrm{b}}$ & $84.9^{\mathrm{b}}$ & 89.9 & $89 \cdot 7$ & $90 \cdot 0$ & $87 \cdot 3^{\mathrm{b}}$ & $89.9^{\mathrm{b}}$ \\
\hline \multicolumn{11}{|l|}{ Basal diet } \\
\hline MG & $93.5^{\mathrm{a}}$ & $91.9^{\mathrm{a}}$ & $93.5^{\mathrm{a}}$ & $95 \cdot 0^{\mathrm{a}}$ & $86 \cdot 5^{\mathrm{a}}$ & $91 \cdot 7^{\mathrm{a}}$ & $92 \cdot 2^{\mathrm{a}}$ & $92 \cdot 7^{\mathrm{a}}$ & $90 \cdot 4^{\mathrm{a}}$ & $92 \cdot 6^{\mathrm{a}}$ \\
\hline PPC & $91.9^{d}$ & $90 \cdot 4^{\mathrm{C}}$ & $92 \cdot 0^{\mathrm{d}}$ & $89 \cdot 9^{\mathrm{c}}$ & $79 \cdot 0^{\mathrm{c}}$ & $88 \cdot 4^{\mathrm{b}}$ & $87.5^{\mathrm{b}}$ & $88 \cdot 9^{\mathrm{b}}$ & $84.4^{\mathrm{d}}$ & $87.5^{\mathrm{C}}$ \\
\hline SFM & $92 \cdot 4^{\mathrm{C}}$ & $90 \cdot 5^{\mathrm{b}}$ & $92 \cdot 7^{\mathrm{c}}$ & $91 \cdot 5^{\mathrm{b}}$ & $87 \cdot 2^{\mathrm{b}}$ & $89.7^{\mathrm{C}}$ & $90 \cdot 6^{\mathrm{b}}$ & $89.8^{\mathrm{b}}$ & $87.8^{\mathrm{C}}$ & $90 \cdot 7^{\mathrm{b}}$ \\
\hline RSM & $89.8^{\mathrm{e}}$ & $89.4^{\mathrm{c}}$ & $91.5^{\mathrm{c}}$ & $89.3^{\mathrm{d}}$ & $84 \cdot 6^{d}$ & $88.5^{\mathrm{d}}$ & $87 \cdot 4^{\mathrm{C}}$ & $87.4^{\mathrm{b}}$ & $84.4^{\mathrm{e}}$ & $87.5^{\mathrm{d}}$ \\
\hline HBM & $93.4^{\mathrm{b}}$ & $90 \cdot 5^{\mathrm{b}}$ & $93 \cdot 2^{\mathrm{b}}$ & $92 \cdot 2^{\mathrm{a}}$ & $87.7^{\mathrm{b}}$ & $90 \cdot 8^{a}$ & $91.0^{\mathrm{b}}$ & $90 \cdot 3^{a}$ & $89 \cdot 6^{\mathrm{b}}$ & $91 \cdot 2^{\mathrm{b}}$ \\
\hline \multicolumn{11}{|c|}{ Means of the diets for the one-way ANOVA model } \\
\hline MG-0 & $93 \cdot 0^{\mathrm{c}, \mathrm{d}}$ & $91 \cdot 3^{\mathrm{c}, \mathrm{d}}$ & $93 \cdot 1^{\mathrm{c}, \mathrm{d}}$ & $94 \cdot 7^{b}$ & $85 \cdot 6^{\mathrm{d}, \mathrm{e}}$ & $91 \cdot 1^{\mathrm{C}}$ & $91.6^{\mathrm{C}}$ & $92 \cdot 1^{\mathrm{c}}$ & $89.8^{\mathrm{c}}$ & $92 \cdot 0^{\mathrm{c}}$ \\
\hline MG-S & $94.0^{\mathrm{b}}$ & $92.5^{\mathrm{b}}$ & $93.9^{b}$ & $95 \cdot 4^{\mathrm{a}, \mathrm{b}}$ & $87 \cdot 3^{b, c}$ & $92 \cdot 4^{\mathrm{b}}$ & $92 \cdot 7^{\mathrm{b}}$ & $93.3^{\mathrm{b}}$ & $91.0^{\mathrm{b}}$ & $93 \cdot 2^{\mathrm{b}}$ \\
\hline PP-0 & $93 \cdot 7^{\mathrm{b}, \mathrm{c}}$ & $91 \cdot 7^{\mathrm{b}, \mathrm{c}}$ & $93 \cdot 4^{\mathrm{c}}$ & $91 \cdot 9^{c, d}$ & $85 \cdot 1^{\mathrm{d}, \mathrm{e}}$ & $91.0^{\mathrm{C}}$ & $90.5^{d}$ & $90 \cdot 4^{d}$ & $88 \cdot 7^{\mathrm{c}, \mathrm{d}}$ & $90 \cdot 3^{\mathrm{d}, \mathrm{e}}$ \\
\hline PP-S & $91.9^{\mathrm{e}}$ & $90 \cdot 4^{\mathrm{e}}$ & $92 \cdot 0^{\dagger}$ & $89.9^{e}$ & $79 \cdot 0^{9}$ & $88.4^{\mathrm{g}}$ & $87 \cdot 5^{e, f}$ & $88.9^{e}$ & $84 \cdot 4^{\mathrm{f}, \mathrm{g}}$ & $87 \cdot 5^{f, g}$ \\
\hline SF-0 & $92 \cdot 4^{\mathrm{d}, \mathrm{e}}$ & $90 \cdot 4^{\mathrm{d}, \mathrm{e}}$ & $92 \cdot 5^{e, f}$ & $91 \cdot 5^{c, d}$ & $87 \cdot 3^{\mathrm{b}, \mathrm{c}}$ & $89 \cdot 6^{\mathrm{e}, \mathrm{f}}$ & $90.5^{\mathrm{d}}$ & $89 \cdot 6^{\mathrm{d}, \mathrm{e}}$ & $87.9^{\mathrm{d}, \mathrm{e}}$ & $90 \cdot 7^{\mathrm{d}, \mathrm{e}}$ \\
\hline SF-S & $92 \cdot 5^{\mathrm{d}, \mathrm{e}}$ & $90 \cdot 6^{\mathrm{d}, \mathrm{e}}$ & $92 \cdot 8^{\mathrm{d}, \mathrm{e}}$ & $91 \cdot 5^{c, d}$ & $87 \cdot 2^{b, c}$ & $89 \cdot 8^{\mathrm{d}, \mathrm{e}, \mathrm{f}}$ & $90 \cdot 6^{c, d}$ & $90 \cdot 1^{d}$ & $87 \cdot 8^{\mathrm{d}, \mathrm{e}}$ & $90 \cdot 7^{\mathrm{d}, \mathrm{e}}$ \\
\hline RS-0 & $90 \cdot 2^{f}$ & $90 \cdot 1^{e}$ & $92 \cdot 0^{\dagger}$ & $89.9^{e}$ & $86 \cdot 0^{c, d}$ & $88 \cdot 9^{\mathrm{f}, \mathrm{g}}$ & $88 \cdot 2^{\mathrm{e}}$ & $87 \cdot 8^{f}$ & $85 \cdot 2^{\dagger}$ & $88.0^{f}$ \\
\hline RS-S & $89.5^{\dagger}$ & $88 \cdot 7^{f}$ & $91 \cdot 0^{9}$ & $88 \cdot 7^{\dagger}$ & $83 \cdot 1^{\dagger}$ & $88 \cdot 1^{9}$ & $86 \cdot 6^{\dagger}$ & $87.0^{f}$ & $83 \cdot 7^{9}$ & $86.9^{9}$ \\
\hline HB-0 & $93 \cdot 4^{\mathrm{b}, \mathrm{c}}$ & $90 \cdot 4^{e}$ & $93 \cdot 2^{c, d}$ & $92 \cdot 3^{\mathrm{c}}$ & $87 \cdot 5^{\mathrm{b}, \mathrm{c}}$ & $90 \cdot 8^{c, d}$ & $91 \cdot 0^{\mathrm{c}, \mathrm{d}}$ & $90 \cdot 1^{d}$ & $89 \cdot 5^{\mathrm{c}}$ & $91 \cdot 3^{\mathrm{c}, \mathrm{d}}$ \\
\hline HB-S & $93 \cdot 4^{b, c}$ & $90 \cdot 6^{\mathrm{d}, \mathrm{e}}$ & $93 \cdot 2^{c, d}$ & $92 \cdot 2^{\mathrm{c}}$ & $87 \cdot 8^{\mathrm{b}}$ & $90 \cdot 9^{c}$ & $90 \cdot 9^{\mathrm{c}, \mathrm{d}}$ & $90.5^{\mathrm{d}}$ & $89.6^{\mathrm{c}}$ & $91 \cdot 1^{\mathrm{c}, \mathrm{d}, \mathrm{e}}$ \\
\hline WG-S & $95 \cdot 4^{\mathrm{a}}$ & $93.9^{\mathrm{a}}$ & $95 \cdot 3^{\mathrm{a}}$ & $95 \cdot 6^{a}$ & $92 \cdot 0^{\mathrm{a}}$ & $94 \cdot 4^{\mathrm{a}}$ & $94.9^{\mathrm{a}}$ & $94.9^{\mathrm{a}}$ & $93.0^{\mathrm{a}}$ & $94 \cdot 8^{\mathrm{a}}$ \\
\hline SBM & $93 \cdot 0^{\mathrm{c}, \mathrm{d}}$ & $90 \cdot 8^{\mathrm{d}, \mathrm{e}}$ & $92.9^{\mathrm{c}, \mathrm{d}, \mathrm{e}}$ & $91 \cdot 3^{\mathrm{d}}$ & $84 \cdot 3^{e, f}$ & $90 \cdot 2^{\mathrm{c}, \mathrm{d}, \mathrm{e}}$ & $90 \cdot 3^{d}$ & $90 \cdot 2^{d}$ & $87 \cdot 2^{\mathrm{e}}$ & $90 \cdot 0^{\mathrm{e}}$ \\
\hline
\end{tabular}

MG, maize gluten; PPC, pea protein concentrate; SFM, sunflower meal; RSM, rapeseed meal; HBM, horsebean meal; -0, non-inclusion; -S, inclusion; PP, pea protein; SF, sunflower; RS, rapeseed; HB, horsebean; WG, wheat gluten; SBM, soyabean meal.

. 
when compared to fish fed MG-0 which had the lowest value. Fish fed HB-0 also had the highest AD for Fe, Mn and $\mathrm{Zn}$, for which SF-0 had the lowest values. For Cu and P, the PP-0 diet had the highest values, whereas SF-0 again ranked the lowest. For $\mathrm{Cu}, \mathrm{Mn}$ and $\mathrm{P}$, there were no significant differences between the basal diets. For Fe, the HB-0 had a significantly higher $\mathrm{AD}$ than the diet with second highest $\mathrm{AD}$, which was RS-0. For Zn, HB-0 was also significantly higher than the two lowest, PP-0 and SF-0.

\section{Retention of nutrients}

Retention of crude protein and energy. Table 10 presents retention values for $\mathrm{CP}$ and energy expressed as percentages of both ingested and digested material. Retention as a percentage of digested material showed greater variance and distinguished the diets less clearly than when expressed as a percentage of ingested material. The retention values were generally high and highest for fish fed the MG-S and WG-S diets.

Saponins did not affect nitrogen and energy retention values significantly, and no significant saponin-basal diet interactions were observed.

Retention of ingested CP differed significantly between the basal diets and was lower for RSM than the other basal diets. A similar trend was discernible for retention of ingested energy and digested CP. For retention of digested energy, the trend was less clear, but the RSM had the lowest values also for digested energy.

Retention of essential amino acids and cysteine. Retention of amino acids showed similar results whether expressed per ingested or digested amino acids. Details for the retention of digested amino acids are presented in Table 11. No values were obtained for retention of Trp. Saponin inclusion showed no significant effect on the retention of any of the amino acids. Moreover, no saponin-basal diet interactions were significant for any of the amino acids.

There were significant differences between basal diets for retention of both ingested (data not shown) and digested methionine, cysteine, arginine, leucine and tyrosine as well as ingested lysine. The highest values were mostly seen for MG and HBM, whereas PPC and RSM had the lowest values. Apparent retention of digested cysteine was the highest among all amino acids, close to $90 \%$ for diets with HBM.

Bile salts and trypsin in intestinal content. Results of analysis of bile salts concentration and trypsin activity in the intestinal content along the intestine are presented in Table 12. Saponins reduced bile salt concentration in fish fed PPC by about $60 \%$ in the PI1, PI 2 and MI sections. For MG and HBM, the effect seemed to be the opposite in PI1 and PI2. In the MI, the pattern seemed to change for MG and HBM towards a negative effect that tended to continue

Table 7. Apparent digestibility of non-essential amino acids and taurine in Atlantic salmon fed the experimental diets (\%)

\begin{tabular}{|c|c|c|c|c|c|c|c|c|c|}
\hline & Ala & Asp-Asn & Cys & Glu-Gln & Gly & Pro & Ser & Tyr & Taurine \\
\hline \multicolumn{10}{|c|}{ Two-way ANOVA model } \\
\hline$P$ (model) & $<0.0001$ & $<0.0001$ & $<0.0001$ & $<0.0001$ & $<0.0001$ & $<0.0001$ & $<0.0001$ & $<0.0001$ & 0.05 \\
\hline Pooled SEM & 0.3 & 0.4 & 0.3 & 0.3 & 0.3 & $0 \cdot 2$ & 0.4 & 0.5 & $4 \cdot 0$ \\
\hline \multicolumn{10}{|c|}{$P$ values effect tests in the two-way ANOVA model } \\
\hline Saponin & 0.01 & 0.01 & 0.80 & 0.09 & 0.12 & 0.05 & 0.0004 & 0.001 & 0.70 \\
\hline Basal diet & $<0.0001$ & $<0.0001$ & $<0.0001$ & $<0.0001$ & $<0.0001$ & $<0.0001$ & $<0.0001$ & $<0.0001$ & 0.01 \\
\hline Interaction & 0.002 & 0.003 & 0.01 & 0.001 & 0.003 & 0.001 & 0.0001 & 0.0001 & 0.56 \\
\hline \multirow{2}{*}{\multicolumn{10}{|c|}{$\begin{array}{l}\text { Marginal means for the two-way } \\
\text { ANOVA model }\end{array}$}} \\
\hline & & & & & & & & & \\
\hline No saponin & $90 \cdot 9^{a}$ & $83 \cdot 2^{a}$ & $73 \cdot 8$ & $92 \cdot 5$ & $82 \cdot 6$ & $86 \cdot 7^{a}$ & $88 \cdot 0^{a}$ & $89 \cdot 5^{\mathrm{a}}$ & $48 \cdot 0$ \\
\hline With saponin & $90 \cdot 4^{\mathrm{b}}$ & $82 \cdot 8^{b}$ & $71 \cdot 6$ & $92 \cdot 0$ & $82 \cdot 1$ & $85 \cdot 6^{b}$ & $87 \cdot 2^{b}$ & $88 \cdot 6^{\mathrm{b}}$ & $47 \cdot 0$ \\
\hline \multicolumn{10}{|l|}{ Basal diet } \\
\hline MG & $93 \cdot 6^{a}$ & $85 \cdot 7^{a}$ & $84 \cdot 9^{a}$ & $94 \cdot 8^{\mathrm{a}}$ & $84.9^{a}$ & $92 \cdot 5^{\mathrm{a}}$ & $91 \cdot 3^{a}$ & $92 \cdot 8^{\mathrm{b}}$ & $50 \cdot 5^{a}$ \\
\hline PPC & $88 \cdot 6^{d}$ & $81.9^{c}$ & $57 \cdot 2^{b}$ & $89 \cdot 9^{c}$ & $79 \cdot 9^{b, c}$ & $81 \cdot 6^{b}$ & $84 \cdot 4^{d}$ & $86 \cdot 4^{d}$ & $38 \cdot 5^{b}$ \\
\hline SFM & $90 \cdot 2^{\mathrm{C}}$ & $81 \cdot 6^{\mathrm{b}}$ & $70 \cdot 3^{b}$ & $92 \cdot 0^{\mathrm{C}}$ & $81 \cdot 4^{c}$ & $85 \cdot 6^{b}$ & $87 \cdot 3^{c}$ & $89 \cdot 2^{a, b}$ & $56 \cdot 9^{a}$ \\
\hline RSM & $88 \cdot 8^{\mathrm{e}}$ & $79 \cdot 0^{d}$ & $69 \cdot 6^{c}$ & $90 \cdot 6^{d}$ & $80 \cdot 4^{\mathrm{d}}$ & $81.9^{c}$ & $84.0^{\mathrm{e}}$ & $85 \cdot 7^{c}$ & $41 \cdot 2^{b}$ \\
\hline HBM & $91 \cdot 2^{\mathrm{b}}$ & $85 \cdot 0^{\mathrm{b}}$ & $75 \cdot 5^{\mathrm{b}}$ & $92 \cdot 7^{\mathrm{b}}$ & $83 \cdot 9^{\mathrm{ab}}$ & $87 \cdot 0^{a}$ & $89 \cdot 1^{\mathrm{b}}$ & $89 \cdot 4^{a}$ & $48 \cdot 6^{a, b}$ \\
\hline \multicolumn{10}{|c|}{ Means of the diets for the one-way ANOVA model } \\
\hline MG-0 & $93 \cdot 1^{\mathrm{b}}$ & $84 \cdot 7^{\mathrm{c}}$ & $83 \cdot 6^{\mathrm{b}}$ & $94 \cdot 4^{\mathrm{b}}$ & $83 \cdot 9^{c}$ & $92 \cdot 0^{\mathrm{b}}$ & $90 \cdot 8^{\mathrm{b}}$ & $92 \cdot 5^{\mathrm{b}}$ & $47 \cdot 3^{\mathrm{b}, \mathrm{c}, \mathrm{d}, \mathrm{e}}$ \\
\hline MG-S & $94 \cdot 0^{\mathrm{a}}$ & $86 \cdot 7^{b}$ & $86 \cdot 2^{b}$ & $95 \cdot 2^{b}$ & $85 \cdot 8^{\mathrm{b}}$ & $92 \cdot 9^{b}$ & $91 \cdot 9^{\mathrm{b}}$ & $93 \cdot 2^{a, b}$ & $53 \cdot 6^{\mathrm{a}, \mathrm{b}, \mathrm{c}, \mathrm{d}}$ \\
\hline PP-0 & $90 \cdot 5^{\mathrm{c}, \mathrm{d}}$ & $85 \cdot 3^{\mathrm{b}, \mathrm{c}}$ & $69 \cdot 1^{\mathrm{d}}$ & $92 \cdot 5^{\mathrm{c}, \mathrm{d}}$ & $83 \cdot 0^{\mathrm{c}, \mathrm{d}}$ & $85 \cdot 8^{d}$ & $88 \cdot 1^{\mathrm{c}, \mathrm{d}}$ & $89 \cdot 9^{c}$ & $42 \cdot 1^{\mathrm{c}, \mathrm{d}, \mathrm{e}}$ \\
\hline PP-S & $88 \cdot 6^{\dagger}$ & $81 \cdot 9^{\mathrm{d}}$ & $57 \cdot 2^{\mathrm{e}}$ & $89.9^{f}$ & $79 \cdot 9^{\mathrm{g}, \mathrm{h}}$ & $81 \cdot 6^{f}$ & $84 \cdot 4^{\mathrm{e}}$ & $86 \cdot 4^{d}$ & $38.5^{\mathrm{e}}$ \\
\hline SF-0 & $90 \cdot 1^{\mathrm{d}, \mathrm{e}}$ & $81 \cdot 5^{d}$ & $70 \cdot 4^{d}$ & $92 \cdot 0^{\mathrm{c}, \mathrm{d}}$ & $81 \cdot 1^{\mathrm{f}, \mathrm{g}}$ & $85 \cdot 7^{d}$ & $87 \cdot 2^{d}$ & $89 \cdot 3^{c}$ & $56 \cdot 1^{a, b, c}$ \\
\hline SF-S & $90 \cdot 2^{\mathrm{d}, \mathrm{e}}$ & $81 \cdot 8^{d}$ & $70 \cdot 2^{d}$ & $92 \cdot 1^{\mathrm{c}, \mathrm{d}}$ & $81 \cdot 6^{\mathrm{d}, \mathrm{e}, \mathrm{f}}$ & $85 \cdot 6^{d}$ & $87 \cdot 4^{d}$ & $89 \cdot 1^{\mathrm{c}}$ & $57 \cdot 7^{\mathrm{a}, \mathrm{b}}$ \\
\hline RS-0 & $89.5^{\mathrm{e}}$ & $79 \cdot 6^{e}$ & $70 \cdot 7^{d}$ & $91.0^{\mathrm{e}}$ & $81 \cdot 4^{e, f}$ & $83 \cdot 3^{\mathrm{e}}$ & $84 \cdot 8^{\mathrm{e}}$ & $86 \cdot 6^{d}$ & $42 \cdot 9^{\mathrm{c}, \mathrm{d}, \mathrm{e}}$ \\
\hline RS-S & $88 \cdot 1^{f}$ & $78 \cdot 3^{e}$ & $68 \cdot 4^{d}$ & $90 \cdot 1^{f}$ & $79 \cdot 3^{h}$ & $80 \cdot 5^{\mathrm{g}}$ & $83 \cdot 2^{f}$ & $84.9^{e}$ & $39 \cdot 5^{\mathrm{d}, \mathrm{e}}$ \\
\hline HB-O & $91 \cdot 1^{\mathrm{C}}$ & $84.9^{c}$ & $75 \cdot 1^{c}$ & $92 \cdot 7^{\mathrm{c}}$ & $83 \cdot 7^{c}$ & $86 \cdot 8^{c}$ & $88 \cdot 9^{c}$ & $89 \cdot 2^{c}$ & $51 \cdot 6^{\mathrm{a}, \mathrm{b}, \mathrm{c}, \mathrm{d}, \mathrm{e}}$ \\
\hline HB-S & $91 \cdot 2^{c}$ & $85 \cdot 1^{b, c}$ & $76 \cdot 0^{c}$ & $92 \cdot 7^{\mathrm{c}}$ & $84 \cdot 2^{c}$ & $87 \cdot 2^{c}$ & $89 \cdot 2^{c}$ & $89 \cdot 5^{c}$ & $45 \cdot 7^{\mathrm{b}, \mathrm{c}, \mathrm{d}, \mathrm{e}}$ \\
\hline WG-S & $93 \cdot 8^{a, b}$ & $88 \cdot 8^{a}$ & $91 \cdot 1^{a}$ & $97 \cdot 4^{\mathrm{a}}$ & $89 \cdot 2^{a}$ & $95 \cdot 0^{a}$ & $93 \cdot 8^{a}$ & $94 \cdot 3^{a}$ & $64 \cdot 6^{\mathrm{a}}$ \\
\hline SBM & $90 \cdot 2^{\mathrm{d}, \mathrm{e}}$ & $83 \cdot 9^{c}$ & $70 \cdot 1^{d}$ & $91 \cdot 7^{\mathrm{d}, \mathrm{e}}$ & $82 \cdot 8^{\mathrm{c}, \mathrm{d}, \mathrm{e}}$ & $85 \cdot 3^{d}$ & $87 \cdot 0^{d}$ & $88 \cdot 6^{c}$ & $50 \cdot 7^{\mathrm{a}, \mathrm{b}, \mathrm{c}, \mathrm{d}, \mathrm{e}}$ \\
\hline
\end{tabular}

MG, maize gluten; PPC, pea protein concentrate; SFM, sunflower meal; RSM, rapeseed meal; HBM, horsebean meal; -0, non-inclusion; -S, inclusion; PP, pea protein; SF, sunflower; RS, rapeseed; HB, horsebean; WG, wheat gluten; SBM, soyabean meal.

a,b,c,d,e,f,g,h Mean values within a column with unlike superscript letters were significantly different $(P<0.05)$. 
Table 8. Apparent digestibility of fatty acids for Atlantic salmon fed the experimental diets (\%)

\begin{tabular}{|c|c|c|c|c|c|c|c|c|c|c|c|c|c|c|c|c|c|}
\hline & SFA & MUFA & $n-3$ PUFA & $n-6$ PUFA & $16: 0$ & $16: 1 n-7$ & $18: 0$ & $18: 1 n-7$ & $18: 1 n-9$ & $18: 1 n-11$ & $18: 2 n-6$ & $18: 3 n-3$ & $20: 1 n-9$ & $20: 2 n-6$ & $22: 1 n-11$ & $20: 5 n-3$ & $22: 6 n-3$ \\
\hline \multicolumn{18}{|c|}{ Two-way ANOVA model } \\
\hline$P($ model $)$ & 0.04 & 0.004 & 0.02 & 0.0002 & 0.05 & 0.001 & 0.02 & 0.02 & 0.002 & 0.0002 & $<0.0001$ & 0.07 & 0.08 & 0.05 & 0.04 & 0.0002 & 0.06 \\
\hline Pooled SEM & $1 \cdot 2$ & 0.2 & 0.2 & 0.2 & $1 \cdot 2$ & 0.1 & 1.6 & 0.3 & 0.3 & 0.1 & 0.1 & 0.2 & 0.3 & 0.5 & 0.4 & 0.1 & 0.3 \\
\hline \multicolumn{18}{|c|}{$P$ values effect tests in the two-way ANOVA model } \\
\hline Saponin & 0.08 & 0.02 & 0.09 & 0.07 & 0.10 & 0.05 & 0.05 & 0.08 & 0.03 & 0.30 & 0.06 & 0.93 & 0.06 & 0.04 & 0.02 & 0.06 & 0.03 \\
\hline Basal diet & 0.02 & 0.001 & 0.01 & $<0.0001$ & 0.03 & 0.0002 & 0.02 & 0.01 & 0.0004 & $<0.0001$ & $<0.0001$ & 0.02 & 0.15 & 0.08 & 0.06 & $<0.0001$ & 0.06 \\
\hline Interaction & 0.22 & 0.18 & 0.30 & 0.51 & 0.22 & 0.21 & 0.13 & 0.47 & 0.16 & 0.94 & 0.41 & 0.31 & 0.11 & 0.11 & 0.11 & 0.41 & 0.27 \\
\hline \multicolumn{18}{|c|}{ Marginal means for the two-way ANOVA model } \\
\hline \multicolumn{18}{|c|}{ Saponin supplementation } \\
\hline No saponin & 87.4 & $98.4^{\mathrm{a}}$ & 98.7 & 98.6 & $87 \cdot 1$ & $98.6^{\mathrm{a}}$ & $83 \cdot 2^{\mathrm{a}}$ & $97 \cdot 7$ & $98.7^{\mathrm{a}}$ & $99 \cdot 3$ & $98 \cdot 7$ & 99.2 & $97 \cdot 4$ & $94.0^{\mathrm{a}}$ & $97 \cdot 1^{\mathrm{a}}$ & $99 \cdot 1$ & $97.6^{\mathrm{a}}$ \\
\hline With saponin & 85.9 & $98 \cdot 1^{\mathrm{b}}$ & 98.4 & 98.4 & $85 \cdot 8$ & $98.4^{\mathrm{b}}$ & $80.9^{\mathrm{b}}$ & $97 \cdot 3$ & $98.5^{\mathrm{b}}$ & 99.3 & 98.6 & 99.2 & $97 \cdot 0$ & $93 \cdot 2^{\mathrm{b}}$ & $96 \cdot 5^{\mathrm{b}}$ & 99.0 & $97 \cdot 1^{\mathrm{b}}$ \\
\hline \multicolumn{18}{|l|}{ Basal diet } \\
\hline MG & $84 \cdot 8^{\mathrm{c}}$ & $98 \cdot 4^{\mathrm{a}, \mathrm{b}}$ & $98 \cdot 8^{\mathrm{a}}$ & $98.9^{\mathrm{a}}$ & $84 \cdot 8^{c}$ & $98 \cdot 8^{\mathrm{a}, \mathrm{b}}$ & $79 \cdot 6^{\mathrm{c}}$ & $97 \cdot 8^{\mathrm{a}, \mathrm{b}}$ & $98 \cdot 9^{a, b}$ & $99.5^{\mathrm{a}}$ & $99.1^{\mathrm{a}}$ & 99.5 & 97.4 & 93.0 & $97 \cdot 1$ & $99 \cdot 3^{\mathrm{a}}$ & $97 \cdot 6$ \\
\hline PPC & $88 \cdot 3^{a, b}$ & $98 \cdot 7^{\mathrm{a}}$ & $98.9^{\mathrm{a}}$ & $99.2^{\mathrm{a}}$ & $88 \cdot 2^{a, b}$ & $99.0^{\mathrm{a}}$ & $84 \cdot 1^{a, b}$ & $98.2^{\mathrm{a}}$ & $99 \cdot 1^{\mathrm{a}}$ & $99.6^{\mathrm{a}}$ & $99 \cdot 3^{\mathrm{a}}$ & 99.6 & 97.6 & 94.1 & 97.4 & $99 \cdot 4^{\mathrm{a}}$ & 97.8 \\
\hline SFM & $85 \cdot 9^{b, c}$ & $97.7^{\mathrm{c}}$ & $98 \cdot 2^{\mathrm{b}}$ & $97.7^{\mathrm{c}}$ & $85 \cdot 6^{\mathrm{b}, \mathrm{c}}$ & $98 \cdot 0^{d}$ & $81 \cdot 3^{b, c}$ & $97 \cdot 2^{\mathrm{b}, \mathrm{c}}$ & $98 \cdot 0^{d}$ & $98.9^{b}$ & $97 \cdot 7^{\circ}$ & 98.9 & $96 \cdot 8$ & 93.0 & $96 \cdot 2$ & $98 \cdot 6^{\mathrm{b}}$ & $96 \cdot 9$ \\
\hline RSM & $85 \cdot 4^{\mathrm{C}}$ & $98 \cdot 2^{b}$ & $98 \cdot 6^{\mathrm{a}, \mathrm{b}}$ & $98.5^{\mathrm{b}}$ & $85 \cdot 2^{c}$ & $98 \cdot 6^{\mathrm{b}, \mathrm{c}}$ & $79 \cdot 9^{c}$ & $96 \cdot 7^{c}$ & $98 \cdot 6^{\mathrm{b}, \mathrm{c}}$ & $99 \cdot 5^{\mathrm{a}}$ & $98 \cdot 6^{\mathrm{b}}$ & 99.1 & 97.1 & 93.3 & 96.7 & $99 \cdot 1^{\mathrm{a}}$ & 97.5 \\
\hline HBM & $88 \cdot 8^{\mathrm{a}}$ & $98 \cdot 1^{\mathrm{b}}$ & $98 \cdot 2^{\mathrm{b}}$ & $98.4^{\mathrm{b}}$ & $88.4^{\mathrm{a}}$ & $98 \cdot 3^{\mathrm{c}}$ & $85 \cdot 6^{\mathrm{a}}$ & $97 \cdot 6^{a, b}$ & $98 \cdot 4^{c}$ & $99 \cdot 1^{\mathrm{b}}$ & $98 \cdot 5^{\mathrm{b}}$ & 98.9 & 96.9 & 94.4 & $96 \cdot 6$ & $98 \cdot 7^{\mathrm{b}}$ & $97 \cdot 0$ \\
\hline \multicolumn{18}{|c|}{ Means of the diets for the one-way ANOVA model } \\
\hline MG-0 & $84.0^{\mathrm{c}}$ & $98.4^{\mathrm{b}}$ & $98 \cdot 7^{a, b, c}$ & $98 \cdot 9^{\mathrm{b}, \mathrm{c}}$ & $84.0^{\mathrm{d}}$ & $98 \cdot 7^{\mathrm{b}, \mathrm{c}}$ & $78.6^{\mathrm{d}}$ & $97.7^{\mathrm{b}}$ & $98 \cdot 8^{b, c, d}$ & $99 \cdot 5^{\mathrm{a}}$ & $99 \cdot 1^{\mathrm{b}, \mathrm{c}}$ & 99.4 & $97 \cdot 3^{\mathrm{a}, \mathrm{b}}$ & $92 \cdot 9^{b, c, d}$ & $97 \cdot 0^{\mathrm{b}, \mathrm{c}}$ & $99 \cdot 3^{a, b}$ & $97 \cdot 6^{\mathrm{a}, \mathrm{b}, \mathrm{c}}$ \\
\hline MG-S & $85.5^{\mathrm{b}, \mathrm{c}}$ & $98.5^{\mathrm{b}}$ & $98 \cdot 9^{a, b}$ & $99 \cdot 0^{\mathrm{a}, \mathrm{b}}$ & $85 \cdot 5^{\mathrm{b}, \mathrm{c}, \mathrm{d}}$ & $98 \cdot 8^{\mathrm{b}}$ & $80 \cdot 6^{\mathrm{c}, \mathrm{d}}$ & $97 \cdot 9^{\mathrm{a}, \mathrm{b}}$ & $98.9^{\mathrm{b}}$ & $99.5^{\mathrm{a}}$ & $99.2^{\mathrm{a}, \mathrm{b}}$ & 99.5 & $97.5^{\mathrm{a}, \mathrm{b}}$ & $93 \cdot 1^{b, c, d}$ & $97 \cdot 3^{\mathrm{a}, \mathrm{b}}$ & $99 \cdot 3^{\mathrm{a}, \mathrm{b}}$ & $97 \cdot 6^{\mathrm{a}, \mathrm{b}, \mathrm{c}}$ \\
\hline PP-0 & $90 \cdot 3^{\mathrm{a}}$ & $99 \cdot 1^{\mathrm{a}}$ & $99 \cdot 3^{\mathrm{a}}$ & $99 \cdot 4^{\mathrm{a}}$ & $90.0^{\mathrm{a}}$ & $99 \cdot 2^{\mathrm{a}}$ & $87 \cdot 4^{\mathrm{a}}$ & $98 \cdot 7^{\mathrm{a}}$ & $99 \cdot 4^{\mathrm{a}}$ & $99 \cdot 6^{\mathrm{a}}$ & $99.5^{\mathrm{a}}$ & 99.7 & $98 \cdot 3^{\mathrm{a}}$ & $95 \cdot 6^{\mathrm{a}}$ & $98 \cdot 2^{\mathrm{a}}$ & $99.5^{\mathrm{a}}$ & $98 \cdot 4^{\mathrm{a}}$ \\
\hline PP-S & $86 \cdot 3^{\mathrm{b}, \mathrm{c}}$ & $98 \cdot 3^{\mathrm{b}}$ & $98 \cdot 6^{\mathrm{b}, \mathrm{c}}$ & $99 \cdot 0^{\mathrm{a}, \mathrm{b}}$ & $86 \cdot 4^{\mathrm{a}, \mathrm{b}, \mathrm{c}, \mathrm{d}}$ & $98 \cdot 7^{\mathrm{b}, \mathrm{c}}$ & $80 \cdot 8^{\mathrm{b}, \mathrm{c}, \mathrm{d}}$ & $97.7^{\mathrm{b}}$ & $98 \cdot 7^{b, c, d}$ & $99 \cdot 5^{a}$ & $99 \cdot 1^{a, b, c}$ & 99.4 & $96.9^{b}$ & $92 \cdot 7^{c, d}$ & $96 \cdot 6^{b, c, d}$ & $99 \cdot 2^{a, b}$ & $97 \cdot 1^{b, c}$ \\
\hline SF-0 & $86 \cdot 9^{a, b, c}$ & $97 \cdot 8^{\mathrm{C}}$ & $98 \cdot 2^{c}$ & $97 \cdot 8^{\mathrm{e}}$ & $86 \cdot 6^{\mathrm{a}, \mathrm{b}, \mathrm{b}, \mathrm{d}}$ & $98 \cdot 1^{\mathrm{de}}$ & $82 \cdot 9^{\mathrm{a}, \mathrm{b}, \mathrm{c}, \mathrm{d}}$ & $97 \cdot 3^{\mathrm{b}, \mathrm{c}}$ & $98 \cdot 1^{e, f}$ & $98 \cdot 9^{\mathrm{b}, \mathrm{c}}$ & $97.8^{f}$ & 98.6 & $96 \cdot 7^{\mathrm{b}}$ & $93 \cdot 3^{\mathrm{b}, \mathrm{c}, \mathrm{d}}$ & $96 \cdot 4^{\mathrm{b}, \mathrm{c}, \mathrm{d}}$ & $98 \cdot 7^{c}$ & $97 \cdot 1^{\mathrm{b}, \mathrm{c}}$ \\
\hline SF-S & $84.9^{c}$ & $97 \cdot 6^{\mathrm{c}}$ & $98 \cdot 2^{\mathrm{c}}$ & $97.6^{\mathrm{e}}$ & $84 \cdot 6^{\mathrm{c}, \mathrm{d}}$ & $98.0^{e}$ & $79.8^{\mathrm{d}}$ & $97.0^{\mathrm{b}, \mathrm{c}}$ & $97.9^{f}$ & $98.8^{\mathrm{c}}$ & $97.7^{f}$ & 99.2 & $96.9^{b}$ & $92 \cdot 7^{\mathrm{c}, \mathrm{d}}$ & $96 \cdot 0^{c, d}$ & $98 \cdot 6^{c}$ & $96 \cdot 8^{\mathrm{c}}$ \\
\hline RS-0 & $86 \cdot 9^{\mathrm{a}, \mathrm{b}, \mathrm{c}}$ & $98 \cdot 5^{\mathrm{b}}$ & $98 \cdot 8^{a, b}$ & $98 \cdot 7^{b, c, d}$ & $86 \cdot 7^{\mathrm{a}, \mathrm{b}, \mathrm{c}, \mathrm{c}, \mathrm{d}}$ & $98 \cdot 7^{\mathrm{b}, \mathrm{c}}$ & $82 \cdot 0^{b, c, d}$ & $97 \cdot 0^{\mathrm{b}, \mathrm{c}}$ & $98 \cdot 9^{\mathrm{b}, \mathrm{c}}$ & $99 \cdot 5^{a}$ & $98 \cdot 8^{\mathrm{b}, \mathrm{c}, \mathrm{d}, \mathrm{e}}$ & $99 \cdot 2$ & $97 \cdot 6^{a, b}$ & $93 \cdot 5^{b, c, d}$ & $97 \cdot 3^{a, b}$ & $99 \cdot 3^{a, b}$ & $97 \cdot 8^{a, b}$ \\
\hline RS-S & $84.0^{\mathrm{C}}$ & $98 \cdot 0^{\mathrm{b}, \mathrm{c}}$ & $98 \cdot 3^{\mathrm{b}, \mathrm{c}}$ & $98 \cdot 3^{\mathrm{d}}$ & $83 \cdot 7^{d}$ & $98 \cdot 4^{\mathrm{b}, \mathrm{c}, \mathrm{d}}$ & $77 \cdot 8^{\mathrm{d}}$ & $96 \cdot 4^{c}$ & $98 \cdot 4^{\mathrm{c}, \mathrm{d}, \mathrm{e}}$ & $99 \cdot 4^{\mathrm{a}}$ & $98.4^{\mathrm{e}}$ & 98.9 & $96 \cdot 6^{\mathrm{b}}$ & $93 \cdot 1^{b, c, d}$ & $96 \cdot 1^{\mathrm{c}, \mathrm{d}}$ & $99 \cdot 0^{\mathrm{b}}$ & $97 \cdot 2^{\mathrm{b}, \mathrm{c}}$ \\
\hline HB-O & $88 \cdot 8^{\mathrm{a}, \mathrm{b}}$ & $98 \cdot 1^{b, c}$ & $98 \cdot 3^{\mathrm{b}, \mathrm{c}}$ & $98 \cdot 4^{c, d}$ & $88 \cdot 3^{a, b, c}$ & $98 \cdot 4^{c, d}$ & $85 \cdot 4^{a, b, c}$ & $97.7^{\mathrm{b}}$ & $98.5^{\mathrm{b}, \mathrm{c}, \mathrm{d}, \mathrm{e}}$ & $99 \cdot 1^{\mathrm{b}}$ & $98 \cdot 5^{\mathrm{d}, \mathrm{e}}$ & 98.9 & $97.0^{\mathrm{b}}$ & $94 \cdot 5^{\mathrm{a}, \mathrm{b}}$ & $96 \cdot 6^{\mathrm{b}, \mathrm{c}, \mathrm{d}}$ & $98 \cdot 7^{\mathrm{c}}$ & $97 \cdot 1^{\mathrm{b}, \mathrm{c}}$ \\
\hline HB-S & $88 \cdot 8^{\mathrm{a}, \mathrm{b}}$ & $98 \cdot 0^{\mathrm{b}, \mathrm{c}}$ & $98 \cdot 1^{c}$ & $98 \cdot 3^{\mathrm{d}}$ & $88 \cdot 6^{\mathrm{a}, \mathrm{b}}$ & $98 \cdot 3^{\mathrm{d}, \mathrm{e}}$ & $85 \cdot 8^{a, b}$ & $97.6^{\mathrm{b}}$ & $98 \cdot 4^{\mathrm{d}, \mathrm{e}, \mathrm{f}}$ & $99 \cdot 0^{\mathrm{b}, \mathrm{c}}$ & $98.4^{e}$ & 98.9 & $96 \cdot 9^{b}$ & $94 \cdot 2^{a, b, c}$ & $96 \cdot 6^{b, c, d}$ & $98 \cdot 6^{c}$ & $96 \cdot 9^{c}$ \\
\hline WG-S & $85 \cdot 4^{\mathrm{b}, \mathrm{c}}$ & $98 \cdot 3^{\mathrm{b}}$ & $98 \cdot 8^{\mathrm{a}, \mathrm{b}}$ & $98 \cdot 6^{b, c, d}$ & $84 \cdot 3^{\mathrm{d}}$ & $98 \cdot 7^{\mathrm{b}, \mathrm{c}}$ & $79 \cdot 1^{d}$ & $97.5^{\mathrm{b}}$ & $98 \cdot 7^{\mathrm{b}, \mathrm{c}, \mathrm{d}}$ & $99.5^{a}$ & $98 \cdot 7^{\mathrm{c}, \mathrm{d}, \mathrm{e}}$ & 99.4 & $97 \cdot 1^{\mathrm{b}}$ & $93 \cdot 0^{\mathrm{b}, \mathrm{c}, \mathrm{d}}$ & $96 \cdot 6^{b, c, d}$ & $99 \cdot 3^{a, b}$ & $97 \cdot 6^{\mathrm{a}, \mathrm{b}, \mathrm{c}}$ \\
\hline SBM & $84 \cdot 4^{\mathrm{c}}$ & $98 \cdot 0^{\mathrm{b}, \mathrm{c}}$ & $98 \cdot 5^{\mathrm{b}, \mathrm{c}}$ & $98 \cdot 8^{b, c, d}$ & $84.0^{\mathrm{d}}$ & $98 \cdot 7^{\mathrm{b}, \mathrm{c}}$ & $77 \cdot 8^{\mathrm{d}}$ & $97 \cdot 2^{b, c}$ & $98 \cdot 5^{\mathrm{b}, \mathrm{c}, \mathrm{d}, \mathrm{e}}$ & $99 \cdot 4^{\mathrm{a}}$ & $98 \cdot 9^{b, c, d}$ & 99.4 & $96 \cdot 6^{\mathrm{b}}$ & $92 \cdot 1^{\mathrm{d}}$ & $95 \cdot 8^{\mathrm{d}}$ & $99 \cdot 2^{\mathrm{b}}$ & $96 \cdot 9^{b, c}$ \\
\hline
\end{tabular}

MG, maize gluten; PPC, pea protein concentrate; SFM, sunflower meal; RSM, rapeseed meal; HBM, horsebean meal; -0, non-inclusion; -S, inclusion; PP, pea protein; SF, sunflower; RS, rapeseed; HB, horsebean; WG, wheat

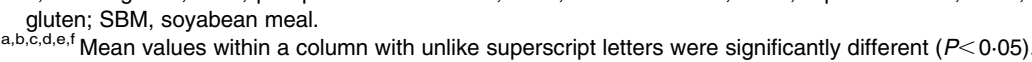


throughout the digestive tract (DI1 and DI2). However, no significant effects of saponins were observed in the two distal sections.

Chyme bile salt concentration in the proximal intestinal sections depended on basal diet, and in MI was highest for MG-0, significantly higher than SF-0 and HB-0. The other basal diets, diet PP-0 and RS-0, showed intermediate bile salt concentrations in MI. This pattern was not clearly reflected in the other intestinal sections.

Saponin supplementation elevated trypsin activity in content from the DI1 and DI2 regions in fish fed PPC. For the other basal diets, there was no significant effect in these sections. No significant saponin effects were seen in the more proximal sections for any of the basal diets. The basal diets, however, differed regarding effects on trypsin activity in content from DI1 and DI2. In DI1, fish fed SF-0 and HB-0 had lower trypsin activity than fish fed MG-0. In the very distal section, DI2, trypsin activity was highest for fish fed PP-0 and significantly higher than for fish fed MG-0, SF-0 and HB-0. Fish fed RS-0 had intermediate values.

The reference SBM diet also induced high trypsin activity in DI1, an activity that was significantly higher than values from other diets except the PP-S diet, which showed an even higher value. Also in the DI2, the highest trypsin activities were recorded for the PP-S diet. However, the SBM was almost as high and not significantly different from the value observed for the PP-S diet. The values observed for PP-S and SBM in the DI2 were several times higher than the lowest value which was observed for the SF-S diet. For all other diets, there was a great drop in trypsin activity between DI1 and DI2.

Brush-border membrane leucine aminopeptidase activity. Activities of LAP in the DI are presented in Table 13, expressed as specific activity and as capacity (activity per kg fish). In the PI and MI sections, no significant effect of saponin supplementation was observed for either the specific activity ( $P$ value/pooled sEm, $0.73 / 44$ for PI and $0 \cdot 28 / 25$ for MI) or capacity ( $P$ value/pooled SEM, $0 \cdot 60 / 39$ for PI and $0 \cdot 09 / 2$ for $\mathrm{MI})$. In DI, saponin inclusion reduced activity expressed both ways for all basal diets, but not to the same degree. The reduction was significant for fish fed PPC and RSM; most pronounced for PPC with a reduction of $74 \%$.

The basal diets did not differ significantly regarding effects on LAP activity in the PI and MI although there was a trend towards higher activity in the MI in fish fed HBM. In the DI, basal diet had significantly different effects on both capacity and specific activity of LAP. The lowest activity was observed in fish fed PPC, intermediate when fed MG, SFM and RSM, and highest when fed HBM.

Organo-somatic index. Saponin numerically reduced OSI of the DI for fish fed all basal diets except MG (Table 14). However, fish fed the PPC were the only ones showing

Table 9. Apparent digestibility coefficients (\%) for selected dietary minerals for Atlantic salmon fed the experimental diets

\begin{tabular}{|c|c|c|c|c|c|c|c|c|c|}
\hline & Ash & $\mathrm{Na}$ & $\mathrm{Ca}$ & $\mathrm{Mg}$ & $\mathrm{Cu}$ & $\mathrm{Fe}$ & $\mathrm{Mn}$ & $\mathrm{Zn}$ & $\mathrm{P}$ \\
\hline \multicolumn{10}{|c|}{ Two-way ANOVA model } \\
\hline$P$ (model) & $<0.0001$ & $<0.0001$ & 0.32 & $<0.0001$ & $<0.0001$ & 0.02 & 0.002 & 0.0004 & 0.04 \\
\hline Pooled SEM & 3.0 & 4.2 & $21 \cdot 2$ & 19.5 & $1 \cdot 7$ & $7 \cdot 0$ & 1.9 & $1 \cdot 7$ & $2 \cdot 3$ \\
\hline \multicolumn{10}{|c|}{$P$ values effect tests in the two-way ANOVA model } \\
\hline Saponin & 0.0001 & $<0.0001$ & 0.19 & 0.001 & 0.001 & 0.98 & 0.32 & 0.53 & 0.71 \\
\hline Basal diet & 0.0003 & $<0.0001$ & 0.29 & $<0.0001$ & $<0.0001$ & 0.01 & 0.0004 & 0.0002 & 0.002 \\
\hline Interaction & $<0.0001$ & $<0.0001$ & 0.41 & 0.04 & 0.04 & 0.05 & 0.05 & 0.003 & 0.03 \\
\hline \multicolumn{10}{|c|}{ Marginal means for the two-way ANOVA model } \\
\hline \multicolumn{10}{|c|}{ Saponin supplementation } \\
\hline No saponin & $-4 \cdot 1^{\mathrm{a}}$ & $42 \cdot 4^{\mathrm{a}}$ & $-13 \cdot 1$ & $-263.6^{a}$ & $27 \cdot 2^{\mathrm{b}}$ & $2 \cdot 7$ & -0.6 & 18.9 & $26 \cdot 8$ \\
\hline With saponin & $-15 \cdot 8^{b}$ & $17 \cdot 6^{\mathrm{b}}$ & $-30 \cdot 8$ & $-314 \cdot 2^{b}$ & $32 \cdot 4^{\mathrm{a}}$ & $2 \cdot 6$ & -1.9 & $19 \cdot 6$ & $26 \cdot 3$ \\
\hline \multicolumn{10}{|l|}{ Basal diet } \\
\hline MG & $-9.7^{\mathrm{b}}$ & $55 \cdot 6^{\mathrm{a}}$ & $-24 \cdot 2$ & $-350 \cdot 2^{c}$ & $37 \cdot 1^{\mathrm{a}}$ & $3 \cdot 6^{\mathrm{b}}$ & $3.3^{\mathrm{a}}$ & $24 \cdot 6^{\mathrm{a}}$ & $32 \cdot 4^{\mathrm{a}}$ \\
\hline PPC & $-17 \cdot 2^{\mathrm{c}}$ & $-13 \cdot 2^{d}$ & $-29 \cdot 3$ & $-371.6^{\mathrm{c}}$ & $36 \cdot 0^{\mathrm{a}}$ & $0.0^{\mathrm{b}}$ & $0 \cdot 3^{\mathrm{a}}$ & $17 \cdot 2^{\mathrm{b}}$ & $29 \cdot 6^{a}$ \\
\hline SFM & $-9.3^{\mathrm{b}}$ & $34 \cdot 3^{\mathrm{b}}$ & $-29 \cdot 3$ & $-274.8^{\mathrm{a}}$ & $15 \cdot 5^{\mathrm{c}}$ & $-13 \cdot 1^{\mathrm{b}}$ & $-8.4^{\mathrm{b}}$ & $13 \cdot 3^{\mathrm{c}}$ & $21 \cdot 8^{\mathrm{b}}$ \\
\hline RSM & $-16 \cdot 9^{c}$ & $21.9^{\mathrm{c}}$ & $-34 \cdot 3$ & $-276 \cdot 0^{b}$ & $28 \cdot 6^{\mathrm{b}}$ & $1.8^{\mathrm{b}}$ & $-4 \cdot 8^{b}$ & $17 \cdot 2^{\mathrm{b}}$ & $20 \cdot 8^{\mathrm{b}}$ \\
\hline HBM & $3 \cdot 3^{\mathrm{a}}$ & $51 \cdot 3^{\mathrm{a}}$ & $7 \cdot 2$ & $-231 \cdot 7^{a}$ & $31 \cdot 6^{b}$ & $21 \cdot 1^{\mathrm{a}}$ & $3 \cdot 3^{\mathrm{a}}$ & $23 \cdot 9^{a}$ & $28 \cdot 2^{\mathrm{a}}$ \\
\hline \multicolumn{10}{|c|}{ Means of the diets for the one-way ANOVA model } \\
\hline MG-0 & $-11 \cdot 3^{\mathrm{c}}$ & $51 \cdot 6^{\mathrm{b}, \mathrm{c}}$ & $-28 \cdot 3$ & $-377 \cdot 4^{\mathrm{c}}$ & $31 \cdot 8^{\mathrm{c}, \mathrm{d}}$ & $-3.5^{\mathrm{c}, \mathrm{d}}$ & $1 \cdot 1^{b, c, d}$ & $21 \cdot 2^{\mathrm{d}}$ & $29 \cdot 6^{\mathrm{b}, \mathrm{c}}$ \\
\hline MG-S & $-8 \cdot 1^{\mathrm{b}, \mathrm{c}}$ & $59 \cdot 7^{\mathrm{a}, \mathrm{b}}$ & $-20 \cdot 0$ & $-323 \cdot 3^{\mathrm{b}, \mathrm{c}}$ & $42 \cdot 4^{\mathrm{b}}$ & $10 \cdot 7^{\mathrm{b}, \mathrm{c}}$ & $5 \cdot 5^{\mathrm{b}}$ & $28 \cdot 1^{\mathrm{b}, \mathrm{c}}$ & $35 \cdot 1^{a, b}$ \\
\hline PP-0 & $6 \cdot 3^{\mathrm{a}}$ & $34 \cdot 7^{\mathrm{d}, \mathrm{e}}$ & $-16 \cdot 1$ & $-246 \cdot 6^{a}$ & $33 \cdot 0^{c, d}$ & $2 \cdot 6^{\mathrm{b}, \mathrm{c}}$ & $2 \cdot 8^{\mathrm{b}, \mathrm{c}, \mathrm{d}}$ & $14 \cdot 9^{e, f}$ & $30 \cdot 6^{a, b, c}$ \\
\hline PP-S & $-40 \cdot 7^{e}$ & $-61 \cdot 0^{\mathrm{g}}$ & $-42 \cdot 6$ & $-496 \cdot 6^{d}$ & $39.0^{\mathrm{b}}$ & $-2 \cdot 6^{c, d}$ & $-2 \cdot 1^{\mathrm{d}, \mathrm{e}}$ & $19 \cdot 5^{\mathrm{d}, \mathrm{e}}$ & $28 \cdot 6^{\mathrm{b}, \mathrm{c}}$ \\
\hline SF-0 & $-9 \cdot 6^{c}$ & $40 \cdot 8^{c, d}$ & -33.9 & $-233 \cdot 5^{a}$ & $11.8^{f}$ & $-22 \cdot 7^{d}$ & $-10 \cdot 3^{f}$ & $12 \cdot 7^{\dagger}$ & $20 \cdot 7^{\mathrm{d}, \mathrm{e}}$ \\
\hline SF-S & $-9 \cdot 1^{c}$ & $27 \cdot 7^{e}$ & $-24 \cdot 8$ & $-206 \cdot 0^{\mathrm{a}}$ & $19 \cdot 2^{\mathrm{e}}$ & $-3 \cdot 5^{c, d}$ & $-6 \cdot 4^{e, f}$ & $13 \cdot 9^{f}$ & $23 \cdot 0^{c, d}$ \\
\hline RS-0 & $-7 \cdot 2^{\mathrm{b}, \mathrm{c}}$ & $32 \cdot 6^{\mathrm{d}, \mathrm{e}}$ & -25.5 & $-247.0^{\mathrm{a}}$ & $29 \cdot 5^{\mathrm{c}, \mathrm{d}}$ & $2 \cdot 7^{\mathrm{b}, \mathrm{c}}$ & $-1 \cdot 3^{\mathrm{c}, \mathrm{d}, \mathrm{e}}$ & $22 \cdot 8^{\mathrm{c}, \mathrm{d}}$ & $26 \cdot 3^{\mathrm{c}, \mathrm{d}}$ \\
\hline RS-S & $-26 \cdot 6^{d}$ & $11 \cdot 1^{f}$ & -43.1 & $-305.0^{\mathrm{b}}$ & $27 \cdot 7^{d}$ & $0.8^{\mathrm{c}}$ & $-8 \cdot 3^{\dagger}$ & $11 \cdot 7^{\dagger}$ & $15 \cdot 2^{\mathrm{e}}$ \\
\hline HB-O & $1 \cdot 1^{a, b}$ & $52 \cdot 2^{b, c}$ & 38.1 & $-223 \cdot 6^{a}$ & $29 \cdot 7^{c, d}$ & $34 \cdot 7^{\mathrm{a}}$ & $4 \cdot 7^{\mathrm{b}, \mathrm{c}}$ & $23 \cdot 0^{c, d}$ & $27 \cdot 0^{c, d}$ \\
\hline HB-S & $5 \cdot 5^{a}$ & $50 \cdot 4^{b, c}$ & -23.7 & $-239.9^{a}$ & $33 \cdot 6^{\mathrm{C}}$ & $7 \cdot 6^{\mathrm{b}, \mathrm{c}}$ & $1 \cdot 9^{b, c, d}$ & $24 \cdot 8^{c, d}$ & $29 \cdot 5^{\mathrm{b}, \mathrm{c}}$ \\
\hline WG-S & $3 \cdot 7^{\mathrm{a}}$ & $71 \cdot 1^{\mathrm{a}}$ & -15.0 & $-229.9^{a}$ & $54 \cdot 3^{a}$ & $23 \cdot 8^{a, b}$ & $13 \cdot 3^{a}$ & $36 \cdot 4^{\mathrm{a}}$ & $37 \cdot 5^{\mathrm{a}}$ \\
\hline SBM & $-7 \cdot 2^{b, c}$ & $23.9^{e}$ & $-31 \cdot 4$ & $-306 \cdot 6^{b}$ & $40 \cdot 3^{b}$ & $19 \cdot 0^{\mathrm{a}, \mathrm{b}, \mathrm{c}}$ & $-1 \cdot 3^{\mathrm{c}, \mathrm{d}, \mathrm{e}}$ & $30 \cdot 7^{b}$ & $25 \cdot 6^{c, d}$ \\
\hline
\end{tabular}

MG, maize gluten; PPC, pea protein concentrate; SFM, sunflower meal; RSM, rapeseed meal; HBM, horsebean meal; -0, non-inclusion; -S, inclusion; PP, pea protein; $\mathrm{SF}$, sunflower; RS, rapeseed; HB, horsebean; WG, wheat gluten; SBM, soyabean meal.

a,b,c,d,e,f,g Mean values within a column with unlike superscript letters were significantly different $(P<0.05)$. 
significant reduction. There was no significant saponin-basal diet interaction.

Significant basal diet differences were discernible for the MI, DI and liver OSI. The OSI for MI in fish fed the HBM was significantly higher than the rest of the basal diets, which did not differ significantly from each other. The DI OSI of fish fed HBM was the highest and was significantly different from PPC and SFM but not RSM and SFM. Fish fed the MG and HBM had the highest liver OSI while fish fed the SFM and PPC diets had the lowest. Intermediate values were observed for fish fed RSM.

OSI for the ST, spleen and kidney showed no apparent effects of either saponin inclusion or composition of the basal diet.

Histology. The scores from the visual analogue scale evaluation of the DI histology are presented in Table 15. In fish fed PPC, saponin inclusion seemed to affect all evaluated histological variables except vacuole size variation. These fish showed higher degrees of mucosal fold fusion, increased width and degree of cellular infiltration of the lamina propria and submucosa, abnormal nucleus position and reduced vacuolisation in enterocytes, and increased numbers of goblet cells. For other basal diets, no saponin effects were observed except for significantly shorter mucosal folds and a wider lamina propria in fish fed RSM and higher numbers of goblet cells in fish fed SFM.

Comparison of the results for the unsupplemented diets did not reveal clear differences between the basal diets for any of the histological parameters.

Plasma parameters. No significant effects of either the saponin inclusion or basal diet were apparent for the plasma indicators of lipid metabolism analysed that included cholesterol, TAG and NEFA (results not presented). Saponin inclusion to most basal diets showed a negative trend on glucose levels $(P=0 \cdot 08$; pooled SEM $=0 \cdot 2$; two-way ANOVA marginal means, without saponin $=5 \cdot 2$, with saponin $=5 \cdot 0$ $\mathrm{mmol} / \mathrm{l}$ ) with the exception of the SFM.

Summary of saponin effects. The effects of saponin supplementation were as follows: For all basal diets, there seemed to be negative effects on AD of lipid and individual fatty acids and on FDM, but to different degrees. For other variables, however, the saponin effects were dependent on the composition of the basal diet, and the predominant picture showed major negative effects for PPC on all, except FER and nutrient retention. For the other basal diets, the effects were minor. There was a trend towards positive effect of saponins

Table 10. Retention of crude protein (CP) and energy in Atlantic salmon fed the experimental diets

\begin{tabular}{|c|c|c|c|c|}
\hline & \multicolumn{2}{|c|}{$\begin{array}{l}\text { Retention \% of } \\
\text { ingested }\end{array}$} & \multicolumn{2}{|c|}{$\begin{array}{l}\text { Retention \% of } \\
\text { digested }\end{array}$} \\
\hline & $\mathrm{CP}$ & Energy & $\mathrm{CP}$ & Energy \\
\hline \multicolumn{5}{|c|}{ Two-way ANOVA model } \\
\hline P (model) & 0.03 & 0.06 & 0.09 & 0.32 \\
\hline Pooled SEM & $2 \cdot 4$ & 3.4 & $2 \cdot 8$ & $4 \cdot 1$ \\
\hline \multicolumn{5}{|c|}{$P$ values effect tests in the two-way ANOVA model } \\
\hline Saponin & 0.22 & 0.54 & 0.19 & $0 \cdot 60$ \\
\hline Basal diet & 0.01 & 0.02 & 0.03 & $0 \cdot 16$ \\
\hline Interaction & 0.57 & 0.36 & 0.63 & 0.50 \\
\hline \multirow{2}{*}{\multicolumn{5}{|c|}{$\begin{array}{l}\text { Marginal means for the two-way ANOVA model } \\
\text { Saponin supplementation }\end{array}$}} \\
\hline & & & & \\
\hline No saponin & $49 \cdot 0$ & $54 \cdot 0$ & $56 \cdot 9$ & $64 \cdot 0$ \\
\hline With saponin & $51 \cdot 0$ & $52 \cdot 6$ & $59 \cdot 4$ & $62 \cdot 6$ \\
\hline \multicolumn{5}{|l|}{ Basal diet } \\
\hline MG & $50 \cdot 1^{a}$ & $59 \cdot 4$ & $56 \cdot 1$ & $66 \cdot 3$ \\
\hline PPC & $52 \cdot 4^{\mathrm{a}}$ & $55 \cdot 0$ & $60 \cdot 8$ & $64 \cdot 6$ \\
\hline SFM & $52 \cdot 8^{\mathrm{a}}$ & $52 \cdot 9$ & $61 \cdot 5$ & $64 \cdot 2$ \\
\hline RSM & $42 \cdot 4^{\mathrm{b}}$ & $44 \cdot 7$ & $51 \cdot 7$ & $56 \cdot 0$ \\
\hline HBM & $52 \cdot 4^{\mathrm{a}}$ & $54 \cdot 5$ & $60 \cdot 5$ & $65 \cdot 2$ \\
\hline \multicolumn{5}{|c|}{ Means of the diets for the one-way ANOVA model } \\
\hline MG-0 & $48 \cdot 1^{a, b, c}$ & $55 \cdot 9^{a, b, c}$ & $54 \cdot 3^{b, c, d}$ & $62 \cdot 8$ \\
\hline MG-S & $52 \cdot 0^{a, b}$ & $62 \cdot 8^{a}$ & $57 \cdot 9^{a, b, c, d}$ & 69.7 \\
\hline PP-0 & $51 \cdot 5^{\mathrm{a}, \mathrm{b}}$ & $58 \cdot 3^{a, b}$ & $59 \cdot 0^{\mathrm{a}, \mathrm{b}, \mathrm{c}, \mathrm{d}}$ & $67 \cdot 6$ \\
\hline PP-S & $53 \cdot 4^{a, b}$ & $51 \cdot 8^{\mathrm{b}, \mathrm{c}, \mathrm{d}}$ & $62 \cdot 7^{\mathrm{a}, \mathrm{b}}$ & $61 \cdot 6$ \\
\hline SF-0 & $53 \cdot 9^{a}$ & $52 \cdot 9^{\mathrm{a}, \mathrm{b}, \mathrm{c}, \mathrm{d}}$ & $62 \cdot 7^{\mathrm{a}, \mathrm{b}}$ & 64.4 \\
\hline SF-S & $51 \cdot 8^{a, b}$ & $52 \cdot 8^{\mathrm{a}, \mathrm{b}, \mathrm{c}, \mathrm{d}}$ & $60 \cdot 2^{\mathrm{a}, \mathrm{b}, \mathrm{c}}$ & 63.9 \\
\hline RS-0 & $42 \cdot 0^{c}$ & $45 \cdot 6^{\mathrm{c}, \mathrm{d}}$ & $50 \cdot 9^{d}$ & $56 \cdot 4$ \\
\hline RS-S & $42 \cdot 8^{\mathrm{C}}$ & $43 \cdot 8^{d}$ & $52 \cdot 4^{\mathrm{c}, \mathrm{d}}$ & $55 \cdot 6$ \\
\hline $\mathrm{HB}-0$ & $49 \cdot 6^{\mathrm{a}, \mathrm{b}, \mathrm{c}}$ & $57 \cdot 2^{a, b}$ & $57 \cdot 4^{\mathrm{a}, \mathrm{b}, \mathrm{c}, \mathrm{d}}$ & 68.5 \\
\hline HB-S & $55 \cdot 2^{\mathrm{a}}$ & $51 \cdot 8^{\mathrm{b}, \mathrm{c}, \mathrm{d}}$ & $63 \cdot 5^{\mathrm{a}}$ & 61.9 \\
\hline WG-S & $55 \cdot 1^{\mathrm{a}}$ & $62 \cdot 7^{\mathrm{a}}$ & $59 \cdot 6^{\mathrm{a}, \mathrm{b}, \mathrm{c}, \mathrm{d}}$ & 68.4 \\
\hline SBM & $45 \cdot 8^{\mathrm{b}, \mathrm{c}}$ & $53 \cdot 2^{\mathrm{a}, \mathrm{b}, \mathrm{c}, \mathrm{d}}$ & $52 \cdot 7^{\mathrm{c}, \mathrm{d}}$ & $61 \cdot 7$ \\
\hline
\end{tabular}

MG, maize gluten; PPC, pea protein concentrate; SFM, sunflower meal; RSM, rapeseed meal; HBM, horsebean meal; -0 , non-inclusion; -S, inclusion; PP, pea protein; SF, sunflower; RS, rapeseed; HB, horsebean; WG, wheat gluten; SBM, soyabean meal.

${ }_{\mathrm{a}, \mathrm{b}, \mathrm{c}, \mathrm{d}}$ Mean values within a column with unlike superscript letters were significantly different $(P<0.05)$. 
on feed intake, significant for SFM, with corresponding results for body weight and TGC. The RSM showed reduced AD of all nutrients, minerals included, when saponins had been added; which was also the case for mineral AD in SFM. Supplementation of saponins resulted in increased $\mathrm{AD}$ of amino acids in MG; reduced brush-border LAP activity in the DI for RSM, with a similar trend for MG, SFM and HBM. Only the PPC showed negative effects of saponin supplementation on growth, trypsin activity in chyme of the DI, and on morphology and histology of the DI.

Summary regarding the basal diets. The basal diet with PPC, as indicated by the PP-0 diet, generally showed high $\mathrm{AD}$ values for all the nutrition variables, with only the $\mathrm{AD}$ of Cys and Trp not ranking highest. Regarding the gut variables, however, PP-0 did less well and some of the variables indicated tendencies towards enteritis-like symptoms. The HB-0 diet overall showed somewhat lower nutritional values than the PP-0, with lower values for protein and amino acid digestibility and protein retention. However, the HB-0 generally showed the best values for many gut variables, but with somewhat reduced bile salt concentration in the intestinal content. The MG-0 diet was intermediate for both the nutrient and gut variables characterised by somewhat lower feed intake and growth, lower AD of Trp, lipid and ash, and CP retention, and some indication of gut reactions. The SF-0 diet showed relatively lower values for all nutrition variables except for the $\mathrm{AD}$ of $\mathrm{Trp}$ and $\mathrm{CP}$ retention, but variables regarding the gut condition were quite good. Overall, the RS-0 showed the lowest values for the $\mathrm{AD}$ variables, and the values for the gut variables also ranked low, but somewhat better than those of the PP-0 diet.

\section{Discussion}

\section{Effects of saponin supplementation}

The negative effect of saponins on lipid digestibility was most probably related to their ability to form insoluble complexes with 3 - $\beta$-hydroxysteroids. They also interact with and form large, mixed micelles with bile salts and cholesterol, accounting for increased bile salt and cholesterol excretion ${ }^{(11,23)}$. The association with bile salts may have implications for lipid digestion and uptake by the intestinal enterocytes. Both a direct effect on lipid emulsification and micelle formation, and an indirect effect secondary to effects on the body pool of bile salts could reduce lipid digestibility.

The observation that saponin supplementation affected feed intake to a variable degree is in agreement with results of other studies. Bureau et al. ${ }^{(12)}$ observed reduced feed intake as an effect of an SBM-derived saponin-rich alcohol extract when fed to Chinook salmon, but not when fed to rainbow trout. Twibell \& Wilson ${ }^{(24)}$ also observed negative saponin

Table 11. Retention of absorbed essential amino acids and cystein in Atlantic salmon fed the experimental diets (\%)

\begin{tabular}{|c|c|c|c|c|c|c|c|c|c|c|}
\hline & Lys & Met & Cys & $\operatorname{Arg}$ & His & lle & Leu & Thr & Phe & Val \\
\hline \multicolumn{11}{|c|}{ Two-way ANOVA model } \\
\hline$P$ (model $)$ & 0.10 & 0.04 & $<0.0001$ & 0.01 & 0.50 & 0.57 & 0.004 & 0.66 & 0.11 & 0.50 \\
\hline Pooled SEM & 3.7 & $3 \cdot 6$ & 4.5 & $2 \cdot 6$ & $3 \cdot 0$ & 3.9 & $2 \cdot 8$ & 3.7 & 3.0 & 4.3 \\
\hline \multicolumn{11}{|c|}{$P$ values effect tests in the two-way ANOVA model } \\
\hline Saponin & 0.33 & 0.23 & 0.67 & 0.14 & 0.32 & 0.20 & 0.23 & 0.45 & 0.51 & 0.58 \\
\hline Basal diet & 0.02 & 0.01 & $<0.0001$ & 0.002 & 0.46 & 0.50 & 0.001 & 0.38 & 0.03 & 0.20 \\
\hline Interaction & 0.70 & 0.53 & 0.32 & 0.48 & 0.45 & 0.66 & 0.60 & 0.82 & 0.70 & 0.87 \\
\hline \multicolumn{11}{|c|}{ Marginal means for the two-way ANOVA model } \\
\hline \multicolumn{11}{|c|}{ Saponin supplementation } \\
\hline No saponin & 57.4 & $55 \cdot 1$ & 72.5 & $44 \cdot 7$ & $48 \cdot 3$ & 49.7 & $44 \cdot 2$ & $57 \cdot 1$ & $46 \cdot 1$ & $50 \cdot 3$ \\
\hline With saponin & $59 \cdot 8$ & $58 \cdot 1$ & 73.8 & $47 \cdot 3$ & $50 \cdot 3$ & 53.0 & $46 \cdot 4$ & $59 \cdot 0$ & 47.4 & $51 \cdot 8$ \\
\hline \multicolumn{11}{|l|}{ Basal diet } \\
\hline MG & 67.5 & $57 \cdot 8^{b}$ & $54 \cdot 3^{b}$ & $55 \cdot 8^{a}$ & $51 \cdot 2$ & $52 \cdot 7$ & $33.0^{b}$ & $58 \cdot 6$ & 41.5 & 53.3 \\
\hline PPC & $55 \cdot 0$ & $50 \cdot 0^{\mathrm{b}}$ & $80 \cdot 2^{\mathrm{a}}$ & $41 \cdot 5^{\mathrm{b}}$ & $49 \cdot 3$ & $51 \cdot 3$ & $49 \cdot 0^{\mathrm{a}}$ & $60 \cdot 2$ & $45 \cdot 8$ & 51.9 \\
\hline SFM & $58 \cdot 2$ & $55 \cdot 6^{\mathrm{b}}$ & $81.9^{\mathrm{a}}$ & $44.6^{\mathrm{b}}$ & 48.4 & $51 \cdot 0$ & $49 \cdot 3^{a}$ & $59 \cdot 1$ & $46 \cdot 2$ & $51 \cdot 3$ \\
\hline RSM & $52 \cdot 9$ & $53 \cdot 1^{\mathrm{b}}$ & $60 \cdot 4^{\mathrm{b}}$ & $44 \cdot 6^{\mathrm{b}}$ & $46 \cdot 3$ & $47 \cdot 4$ & $45 \cdot 2^{\mathrm{a}}$ & 53.1 & $46 \cdot 5$ & 44.0 \\
\hline HBM & $59 \cdot 3$ & $66 \cdot 4^{\mathrm{a}}$ & $89.0^{\mathrm{a}}$ & $43 \cdot 4^{\mathrm{b}}$ & 51.4 & 54.4 & $50 \cdot 0^{\mathrm{a}}$ & $59 \cdot 3$ & $53 \cdot 8$ & 54.7 \\
\hline \multicolumn{11}{|c|}{ Means of the diets for the one-way ANOVA model } \\
\hline MG-0 & $66 \cdot 0^{\mathrm{a}, \mathrm{b}}$ & $55 \cdot 7^{\mathrm{b}, \mathrm{c}, \mathrm{d}}$ & $54 \cdot 6^{\mathrm{c}, \mathrm{d}}$ & $53 \cdot 2^{a, b}$ & 50.5 & 50.9 & $32 \cdot 2^{d}$ & 57.3 & 41.0 & $51 \cdot 8$ \\
\hline MG-S & $69 \cdot 0^{\mathrm{a}}$ & $60 \cdot 0^{\mathrm{a}, \mathrm{b}, \mathrm{c}, \mathrm{d}}$ & $54 \cdot 0^{\mathrm{c}, \mathrm{d}}$ & $58 \cdot 3^{a}$ & $52 \cdot 0$ & 54.5 & $33 \cdot 7^{\mathrm{c}, \mathrm{d}}$ & 59.9 & $42 \cdot 0$ & 54.7 \\
\hline PP-0 & $55 \cdot 4^{\mathrm{b}, \mathrm{c}, \mathrm{d}}$ & $50 \cdot 5^{\mathrm{c}, \mathrm{d}}$ & $82 \cdot 9^{\mathrm{a}, \mathrm{b}}$ & $41 \cdot 8^{\mathrm{C}}$ & $49 \cdot 6$ & 51.5 & $49 \cdot 1^{\mathrm{a}, \mathrm{b}}$ & $60 \cdot 6$ & $46 \cdot 5$ & 53.4 \\
\hline PP-S & $54 \cdot 5^{\mathrm{b}, \mathrm{c}, \mathrm{d}}$ & $49 \cdot 6^{c, d}$ & $77 \cdot 5^{\mathrm{b}}$ & $41 \cdot 1^{c}$ & 49.0 & 51.0 & $48 \cdot 9^{\mathrm{a}, \mathrm{b}}$ & 59.8 & $45 \cdot 0$ & $50 \cdot 4$ \\
\hline SF-0 & $58 \cdot 9^{\mathrm{a}, \mathrm{b}, \mathrm{c}, \mathrm{d}}$ & $57 \cdot 0^{\mathrm{b}, \mathrm{c}, \mathrm{d}}$ & $79 \cdot 7^{b}$ & $45 \cdot 3^{b, c}$ & $49 \cdot 4$ & $51 \cdot 7$ & $49 \cdot 7^{\mathrm{a}, \mathrm{b}}$ & $60 \cdot 1$ & $46 \cdot 6$ & $51 \cdot 7$ \\
\hline SF-S & $57 \cdot 4^{\mathrm{a}, \mathrm{b}, \mathrm{c}, \mathrm{d}}$ & $54 \cdot 2^{\mathrm{b}, \mathrm{c}, \mathrm{d}}$ & $45 \cdot 5^{d}$ & $43 \cdot 9^{c}$ & 47.4 & $50 \cdot 3$ & $48 \cdot 9^{\mathrm{a}, \mathrm{b}}$ & 58.1 & $45 \cdot 7$ & 50.9 \\
\hline RS-0 & $48 \cdot 8^{d}$ & $48 \cdot 7^{d}$ & $58 \cdot 4^{c, d}$ & $41 \cdot 3^{\mathrm{c}}$ & $41 \cdot 7$ & 43.1 & $41 \cdot 5^{b, c}$ & $50 \cdot 2$ & $43 \cdot 2$ & $42 \cdot 2$ \\
\hline RS-S & $57 \cdot 0^{\mathrm{b}, \mathrm{c}, \mathrm{d}}$ & $57 \cdot 4^{\mathrm{b}, \mathrm{c}, \mathrm{d}}$ & $62 \cdot 5^{\mathrm{c}}$ & $47 \cdot 9^{b, c}$ & $50 \cdot 8$ & $51 \cdot 7$ & $48 \cdot 9^{\mathrm{a}, \mathrm{b}}$ & $56 \cdot 1$ & $49 \cdot 8$ & 45.9 \\
\hline HB-O & $57.6^{\mathrm{a}, \mathrm{b}, \mathrm{c}, \mathrm{d}}$ & $63.8^{\mathrm{a}, \mathrm{b}}$ & $82 \cdot 7^{\mathrm{a}, \mathrm{b}}$ & $41 \cdot 7^{\mathrm{C}}$ & $50 \cdot 3$ & 51.3 & $48 \cdot 4^{\mathrm{a}, \mathrm{b}}$ & 57.6 & $53 \cdot 1$ & $52 \cdot 2$ \\
\hline HB-S & $61 \cdot 0^{\mathrm{a}, \mathrm{b}, \mathrm{c}}$ & $69 \cdot 1^{\mathrm{a}}$ & $95 \cdot 2^{\mathrm{a}}$ & $45 \cdot 1^{b, c}$ & 52.4 & 57.6 & $51 \cdot 6^{\mathrm{a}}$ & 61.0 & 54.5 & $57 \cdot 2$ \\
\hline WG-S & $65 \cdot 1^{a, b}$ & $61 \cdot 5^{\mathrm{a}, \mathrm{b}, \mathrm{c}}$ & $77 \cdot 1^{\mathrm{b}}$ & $52 \cdot 3^{a, b}$ & 48.9 & 49.8 & $45 \cdot 5^{\mathrm{a}, \mathrm{b}}$ & $62 \cdot 7$ & 41.0 & 52.2 \\
\hline SBM & $52 \cdot 8^{\mathrm{c}, \mathrm{d}}$ & $56 \cdot 0^{b, c, d}$ & $84 \cdot 2^{\mathrm{a}, \mathrm{b}}$ & $43 \cdot 2^{c}$ & $46 \cdot 5$ & 47.9 & $45 \cdot 7^{\mathrm{a}, \mathrm{b}}$ & $56 \cdot 8$ & $43 \cdot 3$ & $50 \cdot 6$ \\
\hline
\end{tabular}

MG, maize gluten; PPC, pea protein concentrate; SFM, sunflower meal; RSM, rapeseed meal; HBM, horsebean meal; -0, non-inclusion; -S, inclusion; PP, pea protein; SF, sunflower; RS, rapeseed; HB, horsebean; WG, wheat gluten; SBM, soyabean meal.

a,b,c,d Mean values within a column with unlike superscript letters were significantly different $(P<0.05)$. 
Table 12. Bile salt concentration and trypsin activity in intestinal content from the experimental fish

\begin{tabular}{|c|c|c|c|c|c|c|c|c|c|c|}
\hline & \multicolumn{5}{|c|}{ Bile salts (mg/g DM) } & \multicolumn{5}{|c|}{ Trypsin (U/mg DM) } \\
\hline & $\mathrm{Pl} 1$ & $\mathrm{Pl} 2$ & Ml & DI1 & $\mathrm{DI} 2$ & $\mathrm{Pl} 1$ & $\mathrm{Pl} 2$ & $\mathrm{Ml}$ & DI1 & DI2 \\
\hline \multicolumn{11}{|c|}{ Two-way ANOVA model } \\
\hline $\mathrm{P}$ (model) & 0.02 & 0.11 & 0.01 & 0.21 & 0.23 & 0.51 & 0.19 & 0.20 & $<0.0001$ & $<0.0001$ \\
\hline Pooled SEM & 15 & 13 & 9 & 7 & 2 & 46 & 23 & 27 & 9 & 7 \\
\hline \multicolumn{11}{|c|}{$P$ values effect tests in the two-way ANOVA model } \\
\hline Saponin & 0.24 & 0.29 & 0.003 & 0.02 & 0.02 & 0.75 & 0.46 & 0.52 & 0.59 & 0.0003 \\
\hline Basal diet & 0.17 & 0.27 & 0.03 & 0.65 & 0.32 & 0.48 & 0.45 & 0.06 & $<0.0001$ & $<0.0001$ \\
\hline Interaction & 0.01 & 0.06 & 0.02 & 0.40 & 0.86 & 0.36 & 0.08 & 0.71 & 0.0002 & $<0.0001$ \\
\hline \multirow{2}{*}{\multicolumn{11}{|c|}{ Marginal means for the two-way ANOVA model }} \\
\hline & & & & & & & & & & \\
\hline No saponin & 146 & 131 & $116^{\mathrm{a}}$ & 45 & 14 & 248 & 183 & 175 & 64 & $18^{\mathrm{b}}$ \\
\hline With saponin & 135 & 122 & $94^{\mathrm{b}}$ & 33 & 9 & 238 & 172 & 164 & 61 & $35^{\mathrm{a}}$ \\
\hline \multicolumn{11}{|l|}{ Basal diet } \\
\hline$M G$ & 148 & 127 & $126^{\mathrm{a}}$ & 44 & 13 & 282 & 203 & 230 & $54^{\mathrm{b}, \mathrm{c}}$ & $11^{\mathrm{b}}$ \\
\hline PPC & 125 & 109 & $89^{b}$ & 38 & 14 & 224 & 170 & 160 & $106^{\mathrm{a}}$ & $87^{\mathrm{a}}$ \\
\hline SFM & 159 & 138 & $102^{b}$ & 40 & 10 & 261 & 180 & 143 & $48^{c}$ & $9^{b}$ \\
\hline RSM & 126 & 125 & $102^{b}$ & 38 & 10 & 248 & 176 & 167 & $71^{\mathrm{b}}$ & $17^{\mathrm{b}}$ \\
\hline HBM & 145 & 132 & $105^{\mathrm{b}}$ & 33 & 11 & 201 & 160 & 148 & $35^{c}$ & $10^{\mathrm{b}}$ \\
\hline \multicolumn{11}{|c|}{ Means of the diets for the one-way ANOVA model } \\
\hline MG-0 & $138^{a, b, c}$ & 117 & $139^{a}$ & 54 & $16^{a, b}$ & 242 & 190 & 254 & $87^{c}$ & $13^{c}$ \\
\hline MG-S & $159^{a, b}$ & 137 & $114^{\mathrm{a}, \mathrm{b}, \mathrm{c}}$ & 33 & $10^{b, c, d}$ & 321 & 216 & 205 & $21^{\dagger}$ & $8^{\mathrm{C}}$ \\
\hline PP-0 & $177^{\mathrm{a}}$ & 137 & $124^{\mathrm{a}, \mathrm{b}}$ & 48 & $15^{\mathrm{b}, \mathrm{c}}$ & 277 & 188 & 176 & $70^{\mathrm{c}, \mathrm{d}}$ & $37^{\mathrm{b}}$ \\
\hline PP-S & $72^{d}$ & 80 & $54^{d}$ & 28 & $13^{b, c, d}$ & 170 & 151 & 144 & $141^{\mathrm{a}}$ & $136^{\mathrm{a}}$ \\
\hline SF-0 & $156^{a, b}$ & 147 & $108^{\mathrm{b}, \mathrm{c}}$ & 49 & $13^{b, c, d}$ & 260 & 191 & 144 & $58^{\mathrm{d}, \mathrm{e}}$ & $12^{\mathrm{c}}$ \\
\hline SF-S & $161^{a, b}$ & 128 & $97^{\mathrm{b}, \mathrm{c}}$ & 32 & $7^{\mathrm{d}}$ & 262 & 170 & 142 & $38^{e, f}$ & $5^{\mathrm{c}}$ \\
\hline RS-0 & $129^{\mathrm{a}, \mathrm{b}, \mathrm{c}}$ & 130 & $110^{\mathrm{a}, \mathrm{b}, \mathrm{c}}$ & 40 & $12^{\mathrm{b}, \mathrm{c}, \mathrm{d}}$ & 243 & 216 & 156 & $73^{\mathrm{c}, \mathrm{d}}$ & $21^{b, c}$ \\
\hline RS-S & $122^{b, c}$ & 120 & $95^{\mathrm{b}, \mathrm{c}}$ & 36 & $8^{c, d}$ & 254 & 135 & 179 & $70^{\mathrm{c}, \mathrm{d}}$ & $13^{\mathrm{c}}$ \\
\hline HB-O & $131^{a, b, c}$ & 121 & $100^{\mathrm{b}, \mathrm{c}}$ & 33 & $12^{\mathrm{b}, \mathrm{c}, \mathrm{d}}$ & 170 & 131 & 147 & $34^{e, f}$ & $8^{\mathrm{c}}$ \\
\hline HB-S & $159^{\mathrm{a}, \mathrm{b}}$ & 143 & $109^{\mathrm{a}, \mathrm{b}, \mathrm{c}}$ & 34 & $9^{b, c, d}$ & 231 & 189 & 149 & $37^{e, f}$ & $11^{\mathrm{c}}$ \\
\hline WG-S & $103^{c, d}$ & 103 & $94^{b, c}$ & 42 & $22^{\mathrm{a}}$ & 174 & 133 & 197 & $65^{\mathrm{c}, \mathrm{d}}$ & $22^{b, c}$ \\
\hline SBM & $117^{\mathrm{b}, \mathrm{c}, \mathrm{d}}$ & 100 & $88^{\mathrm{c}}$ & 31 & $11^{\mathrm{b}, \mathrm{c}, \mathrm{d}}$ & 280 & 227 & 187 & $115^{\mathrm{b}}$ & $129^{a}$ \\
\hline
\end{tabular}

PI, pyloric intestine; MI, mid-intestine; DI, distal intestine; MG, maize gluten; PPC, pea protein concentrate; SFM, sunflower meal; RSM, rapeseed meal; HBM, horsebean meal; -0, non-inclusion; -S, inclusion; PP, pea protein; SF, sunflower; RS, rapeseed; HB, horsebean; WG, wheat gluten; SBM, soyabean meal.

a,b,c,d,e, $\mathrm{M}$ Mean values within a column with unlike superscript letters were significantly different $(P<0.05)$. 
effects on juvenile channel catfish (Ictalurus punctatus). However, the results of Knudsen et al. ${ }^{(6,7)}$ showed no effects on feed intake when supplemented to diets with lupin meal. It may be suggested that the varying effects of saponin supplementation are due to varying doses of saponins in the diets, being positive at low levels and negative at higher levels ${ }^{(24)}$. However, our chances of evaluating this suggestion are hampered by lack of solid information on saponin content of various plant ingredients. No standardised methods for quantification of saponins are available, and reliability and reproducibility of the methods in use are not satisfactory.

The mechanism behind the negative effects of saponin supplementation to the diets with PPC and to some degree to the diet with RSM on nutrient digestibility is not clear, but interactions with one or more of the many ANF are likely. Several ANF are present in peas including protease inhibitors, phytic acid, oligosaccharides, lectins, tannins and saponins ${ }^{(25,26)}$. Many of the same types are found in RS which are characterised by a very high fibre and phytate content, presence of condensed tannins, a potent trypsin inhibitor, anthocyanidins, glucosinolates and lectins ${ }^{(27-29)}$. All, possibly except the glucosinolates, have the potential to interfere with digestive processes. However, hardly any information exists regarding interactions between antinutrients and effects on digestion.
Questions regarding their interaction with saponins cannot be answered without further investigations.

The negative effect of saponin inclusion on $\mathrm{AD}$ of $\mathrm{Na}$ in the PPC, SFM and RSM diets, and for Mg in PPC and RSM basal diets may also have been the result of complex formation between the ANF of these protein sources and minerals in the intestinal lumen, effects on intestinal uptake mechanisms, or on mineral recirculation between the intestine and the body. $\mathrm{Na}$ uptake in marine fish is a key in osmoregulation and has been demonstrated to occur along the entire GIT but more significantly in the anterior segments (reviewed by Grosell $\left.^{(30)}\right)$. However, the magnitude of the alterations, and the fact that many of the values were negative, indicate that altered drinking behaviour may have been a major factor in these effects. The low FDM in the PP-S and the SBM diets suggests that the fish fed these diets had diarrhoea, which could, at least partly, explain the lower Na AD. The difference in $\mathrm{AD}$ of $\mathrm{Na}$ between the PP-0 and PP-S diets was remarkable (35\% compared to $-61 \%$ ), and supports this suggestion. However, the fact that the $\mathrm{Na} \mathrm{AD}$ in fish fed the PP-0 diet, which did not show low FDM, was not different from that of SBM, indicates that other mechanisms are involved. The positive effect of saponin supplementation on $\mathrm{AD}$ of $\mathrm{Cu}, \mathrm{FE}$, $\mathrm{Mn}, \mathrm{Zn}$ and $\mathrm{P}$ for the MG and SFM diets may be related to

Table 13. Leucine aminopeptidase activity in intestinal tissue homogenates from pyloric, mid and distal intestine from Atlantic salmon fed the experimental diets

\begin{tabular}{|c|c|c|c|c|c|c|}
\hline & \multicolumn{3}{|c|}{$\begin{array}{l}\text { Total capacity (mM/kg BW } \\
\text { (mmol/h per kg fish weight) }\end{array}$} & \multicolumn{3}{|c|}{$\begin{array}{l}\text { Specific activity, } \mathrm{mm} / \mathrm{g} \text { protein } \\
(\mu \mathrm{mol} / \mathrm{h} \text { per } \mathrm{mg} \text { tissue protein) }\end{array}$} \\
\hline & Pyloric & Mid & Distal & Pyloric & Mid & Distal \\
\hline \multicolumn{7}{|c|}{ Two-way ANOVA model } \\
\hline$P$ (model) & 0.60 & 0.09 & 0.0003 & 0.73 & 0.28 & $<0.0001$ \\
\hline Pooled SEM & 39 & 2 & 9 & 44 & 25 & 26 \\
\hline \multicolumn{7}{|c|}{$P$ values effect tests in the two-way ANOVA model } \\
\hline Saponin & 0.72 & 0.46 & 0.0004 & 0.61 & 0.90 & 0.0002 \\
\hline Basal diet & 0.57 & 0.02 & 0.0001 & 0.39 & 0.58 & 0.0001 \\
\hline Interaction & 0.69 & 0.49 & 0.29 & 0.49 & 0.43 & 0.01 \\
\hline \multicolumn{7}{|c|}{ Marginal means for the two-way ANOVA model } \\
\hline \multicolumn{7}{|c|}{ Saponin supplementation } \\
\hline No saponin & 275 & 17 & $95^{\mathrm{a}}$ & 357 & 223 & $409^{\mathrm{a}}$ \\
\hline With saponin & 266 & 18 & $66^{\mathrm{b}}$ & 342 & 225 & $314^{\mathrm{b}}$ \\
\hline \multicolumn{7}{|l|}{ Basal diet } \\
\hline MG & 257 & 17 & $77^{\mathrm{b}}$ & 322 & 243 & $352^{\mathrm{c}}$ \\
\hline PPC & 276 & 19 & $39^{\mathrm{c}}$ & 351 & 234 & $227^{d}$ \\
\hline SFM & 247 & 16 & $85^{\mathrm{b}}$ & 329 & 234 & $376^{\mathrm{b}, \mathrm{c}}$ \\
\hline RSM & 265 & 13 & $91^{a, b}$ & 367 & 231 & $414^{\mathrm{a}, \mathrm{b}}$ \\
\hline HBM & 309 & 21 & $110^{\mathrm{a}}$ & 378 & 178 & $437^{\mathrm{a}}$ \\
\hline \multicolumn{7}{|c|}{ Means of the diets for the one-way ANOVA model } \\
\hline MG-0 & 261 & 14 & $83^{\mathrm{b}, \mathrm{c}}$ & 342 & 238 & $369^{b, c}$ \\
\hline MG-S & 253 & 19 & $70^{\mathrm{b}, \mathrm{c}, \mathrm{d}}$ & 303 & 249 & $336^{\mathrm{b}, \mathrm{c}}$ \\
\hline PP-0 & 312 & 19 & $63^{c, d}$ & 402 & 240 & $360^{\mathrm{b}, \mathrm{c}}$ \\
\hline PP-S & 241 & 19 & $13^{\mathrm{e}}$ & 299 & 229 & $94^{d}$ \\
\hline SF-0 & 225 & 16 & $396^{\mathrm{a}, \mathrm{b}}$ & 303 & 216 & $393^{a, b, c}$ \\
\hline SF-S & 268 & 17 & $74^{b, c, d}$ & 355 & 252 & $358^{\mathrm{b}, \mathrm{c}}$ \\
\hline RS-0 & 271 & 15 & $110^{\mathrm{a}}$ & 360 & 258 & $458^{\mathrm{a}}$ \\
\hline RS-S & 258 & 12 & $71^{\mathrm{b}, \mathrm{c}, \mathrm{d}}$ & 374 & 204 & $371^{\mathrm{b}, \mathrm{c}}$ \\
\hline HB-0 & 307 & 20 & $122^{\mathrm{a}}$ & 376 & 165 & $465^{\mathrm{a}}$ \\
\hline HB-S & 310 & 23 & $98^{\mathrm{a}, \mathrm{b}}$ & 379 & 192 & $409^{a, b}$ \\
\hline WG-S & 224 & 16 & $53^{d}$ & 302 & 209 & $311^{\mathrm{c}}$ \\
\hline SBM & 288 & 14 & $20^{e}$ & 364 & 189 & $129^{d}$ \\
\hline
\end{tabular}

BW, body weight; MG, maize gluten; PPC, pea protein concentrate; SFM, sunflower meal; RSM, rapeseed meal; HBM, horsebean meal; -0, non-inclusion; -S, inclusion; PP, pea protein; SF, sunflower; RS, rapeseed; HB, horsebean; WG, wheat gluten; SBM, soyabean meal.

a,b,c,d,e Mean values within a column with unlike superscript letters were significantly different $(P<0.05)$. 
Table 14. Organo-somatic indices (SI) from the Atlantic salmon fed the experimental diets*

\begin{tabular}{|c|c|c|c|c|c|c|c|c|}
\hline & \multirow[t]{2}{*}{ Stomach SI } & \multicolumn{3}{|c|}{ Intestine SI } & \multirow[t]{2}{*}{ Liver SI } & \multirow[t]{2}{*}{ Spleen SI } & \multicolumn{2}{|c|}{ Kidney SI } \\
\hline & & Pyloric & Mid & Distal & & & Trunk & Head \\
\hline \multicolumn{9}{|c|}{ Two-way ANOVA model } \\
\hline$P($ model $)$ & 0.35 & 0.49 & 0.0004 & 0.01 & 0.02 & 0.19 & 0.94 & 0.44 \\
\hline Pooled SEM & 0.02 & 0.13 & 0.01 & 0.03 & 0.05 & 0.01 & 0.02 & 0.01 \\
\hline \multicolumn{9}{|c|}{$P$ values effect tests in the two-way ANOVA model } \\
\hline Saponin & 0.32 & 0.80 & 0.32 & 0.05 & 0.75 & 0.54 & 0.50 & 0.25 \\
\hline Basal diet & 0.12 & 0.14 & $<0.0001$ & 0.003 & 0.004 & 0.05 & 0.83 & 0.51 \\
\hline Interaction & 0.91 & 0.99 & 0.17 & 0.20 & 0.64 & 0.73 & 0.88 & 0.36 \\
\hline \multicolumn{9}{|c|}{ Marginal means for the two-way ANOVA model } \\
\hline \multicolumn{9}{|c|}{ Saponin supplementation } \\
\hline No saponin & 0.48 & 1.93 & 0.22 & $0.51^{\mathrm{a}}$ & $1 \cdot 22$ & 0.08 & 0.55 & 0.14 \\
\hline With saponin & 0.49 & 1.95 & 0.22 & $0.47^{\mathrm{b}}$ & $1 \cdot 21$ & 0.08 & 0.56 & 0.14 \\
\hline \multicolumn{9}{|l|}{ Basal diet } \\
\hline MG & 0.47 & $2 \cdot 16$ & $0.20^{\mathrm{b}}$ & $0.48^{\mathrm{b}}$ & $1 \cdot 28^{\mathrm{a}, \mathrm{b}}$ & $0 \cdot 10$ & 0.54 & 0.14 \\
\hline PPC & 0.49 & 1.87 & $0.21^{\mathrm{b}}$ & $0.40^{\mathrm{C}}$ & $1 \cdot 16^{\mathrm{c}}$ & 0.08 & 0.56 & 0.13 \\
\hline SFM & 0.47 & 1.86 & $0.21^{\mathrm{b}}$ & $0.51^{\mathrm{b}}$ & $1.11^{\mathrm{c}}$ & 0.07 & 0.55 & 0.14 \\
\hline RSM & 0.46 & 1.81 & $0.19^{b}$ & $0.49^{b}$ & $1 \cdot 17^{\mathrm{b}, \mathrm{c}}$ & 0.08 & 0.56 & 0.15 \\
\hline HBM & 0.52 & $2 \cdot 00$ & $0 \cdot 29^{a}$ & $0.57^{\mathrm{a}}$ & $1 \cdot 35^{a}$ & 0.08 & 0.55 & 0.13 \\
\hline \multicolumn{9}{|c|}{ Means of the diets for the one-way ANOVA model } \\
\hline MG-0 & 0.47 & $2 \cdot 16$ & $0 \cdot 19^{b}$ & $0.46^{\mathrm{b}, \mathrm{c}}$ & $1 \cdot 27^{\mathrm{a}, \mathrm{b}, \mathrm{c}, \mathrm{d}}$ & $0 \cdot 10$ & 0.53 & 0.137 \\
\hline MG-S & 0.47 & $2 \cdot 15$ & $0.21^{b}$ & $0.49^{a, b, c}$ & $1 \cdot 29^{\mathrm{a}, \mathrm{b}, \mathrm{c}}$ & 0.10 & 0.56 & 0.149 \\
\hline PP-0 & 0.49 & 1.85 & $0.20^{\mathrm{b}}$ & $0.46^{\mathrm{b}, \mathrm{c}}$ & $1 \cdot 19^{b, c, d}$ & 0.08 & 0.57 & 0.135 \\
\hline PP-S & 0.49 & 1.89 & $0.22^{\mathrm{b}}$ & $0.35^{\mathrm{d}}$ & $1 \cdot 13^{\mathrm{d}, \mathrm{c}}$ & 0.09 & 0.56 & 0.132 \\
\hline SF-0 & 0.46 & 1.88 & $0.22^{b}$ & $0.52^{a, b}$ & $1 \cdot 13^{\mathrm{d}, \mathrm{c}}$ & 0.07 & 0.55 & 0.142 \\
\hline SF-S & 0.48 & 1.85 & $0.20^{\mathrm{b}}$ & $0.50^{\mathrm{a}, \mathrm{b}, \mathrm{c}}$ & $1.09^{d}$ & 0.08 & 0.55 & 0.136 \\
\hline RS-0 & 0.45 & 1.79 & $0.20^{\mathrm{b}}$ & $0.54^{\mathrm{a}, \mathrm{b}}$ & $1 \cdot 19^{b, c, d}$ & 0.08 & 0.56 & 0.159 \\
\hline RS-S & 0.47 & 1.82 & $0 \cdot 19^{\mathrm{b}}$ & $0.45^{\mathrm{b}, \mathrm{c}}$ & $1 \cdot 16^{\mathrm{b}, \mathrm{c}, \mathrm{d}}$ & 0.08 & 0.57 & 0.133 \\
\hline HB-0 & 0.50 & 1.96 & $0.28^{a}$ & $0.58^{a}$ & $1 \cdot 32^{a, b}$ & 0.08 & 0.54 & 0.136 \\
\hline HB-S & 0.54 & 2.05 & $0.30^{\mathrm{a}}$ & $0.57^{\mathrm{a}}$ & $1 \cdot 39^{a}$ & 0.09 & 0.56 & 0.128 \\
\hline WG-S & 0.43 & 2.06 & $0.22^{b}$ & $0.42^{\mathrm{c}, \mathrm{d}}$ & $1 \cdot 22^{\mathrm{b}, \mathrm{c}, \mathrm{d}}$ & 0.10 & 0.54 & 0.140 \\
\hline SBM & 0.47 & 2.02 & $0 \cdot 21^{b}$ & $0.41^{\mathrm{c}, \mathrm{d}}$ & $1 \cdot 18^{b, c, d}$ & 0.09 & 0.55 & 0.138 \\
\hline
\end{tabular}

MG, maize gluten; PPC, pea protein concentrate; SFM, sunflower meal; RSM, rapeseed meal; HBM, horsebean meal; -0, non-inclusion; -S, inclusion; PP, pea protein; SF, sunflower; RS, rapeseed; HB, horsebean; WG, wheat gluten; SBM, soyabean meal.

$a, b, c, d$ Mean values within a column with unlike superscript letters were significantly different $(P<0.05)$.

* Organ weight as a percentage of fish body weight.

an increase in permeability of the intestine ${ }^{(7,10,31)}$ which has been an observed effect of soyasaponins.

A mechanism for the positive effect of saponins in the MG diet on $\mathrm{AD}$ of amino acids may be related to stimulatory effects of saponins on trypsin activity in the intestinal chyme. Although not significant, there was a numerical increase in the activity of trypsin in PI1 of $30 \%$, and the activity was elevated also in PI2. Elevated trypsin activities were observed also for HBM, but without the beneficial effect on amino acid AD. The explanation for the difference is possibly related to ANF in the HBM that may have hindered a beneficial effect.

The negative effects of saponins on LAP activity of the distal intestinal brush border of fish for all basal diets, significant for PPC and RSM, are in agreement with results of an earlier study with Atlantic salmon ${ }^{(32)}$ in which effects of the same saponin preparation, supplemented at 1 and $2 \mathrm{~g}$ per $\mathrm{kg}$ in a diet with $30 \%$ soya protein concentrate were investigated. The explanation for this reduction in enzyme activity can only be speculative as current knowledge is weak. Saponins have many recorded biological and pharmacological activities and a large number of them are ascribed to their cell membrane effects $^{(7)}$ which may affect BBM enzymes directly or indirectly by increasing cell proliferation. The effects on LAP may have been attributed to a loss of differentiated intestinal epithelial cells and their subsequent replacement with immature cells expressing less BBM enzymes ${ }^{(19)}$.

Supplementation of saponins to the basal diet with PPC caused effects in addition to those shared by one or more of the other basal diets: negative effect on growth, elevation of trypsin activity of chyme in the distal intestinal compartments and enteritis in the DI. These clear disruptive effects were very similar to the symptoms typical for soyabean-induced enteritis observed also in fish fed the SBM. In a recent study, similar histological observations were seen in fish fed a diet with somewhat higher PPC inclusion (35\%) compared to the present work ${ }^{(20)}$ and without saponin supplementation. This indicates that the saponin level in PPC could be high enough to induce enteritis when the dietary inclusion level is high or when combined with SBM or other saponin-containing ingredients. Whether saponins can cause these effects alone is a matter of discussion. There is a possibility that development of enteritis requires another alcohol-soluble component in addition to the saponins, a component that is present in soyabeans, peas, lupins and possibly other legumes. This was also suggested by Knudsen et al. ${ }^{(7)}$ based on information gained from saponin supplementation of a diet with lupin kernel meal, using a $65 \%$ purified soyasaponin extract, which induced enteritis. The work of Knudsen et al. ${ }^{(7)}$ showed a dose-dependency of the saponin effects. In the present 
Table 15. Details of the score-based evaluation of the intestinal histology of fish fed the experimental diets*

\begin{tabular}{|c|c|c|c|c|c|c|c|c|c|c|c|}
\hline & \multicolumn{2}{|c|}{ Mucosal folds } & \multicolumn{2}{|c|}{ Lamina propria } & \multicolumn{2}{|c|}{ Submucosa } & \multicolumn{3}{|c|}{ Enterocytes } & \multicolumn{2}{|c|}{ Other cell types } \\
\hline & Height & Fusion & Width & $\begin{array}{l}\text { Cellularity } \\
\text { infiltration }\end{array}$ & Width & $\begin{array}{l}\text { Cellularity } \\
\text { infiltration }\end{array}$ & $\begin{array}{l}\text { Nuclear } \\
\text { position }\end{array}$ & $\begin{array}{l}\text { Vacuole } \\
\text { size }\end{array}$ & $\begin{array}{l}\text { Vacuole size } \\
\text { variation }\end{array}$ & $\begin{array}{c}\mathrm{IEL} \\
\text { numbers }\end{array}$ & $\begin{array}{c}\text { Goblet cell } \\
\text { numbers }\end{array}$ \\
\hline \multicolumn{12}{|c|}{ Two-way ANOVA model } \\
\hline$P$ (model) & 0.01 & 0.003 & $<0.0001$ & $<0.0001$ & 0.02 & 0.01 & 0.001 & 0.0002 & 0.13 & 0.45 & 0.001 \\
\hline Pooled SEM & 0.5 & 0.7 & 0.3 & 0.4 & 0.4 & 0.5 & 0.5 & 0.3 & 0.1 & 0.8 & 0.6 \\
\hline \multicolumn{12}{|c|}{$P$ values effect tests in the two-way ANOVA model } \\
\hline Saponin & 0.01 & 0.003 & $<0.0001$ & 0.0001 & 0.06 & 0.01 & 0.001 & 0.001 & 0.68 & 0.15 & 0.0003 \\
\hline Basal diet & 0.01 & 0.01 & $<0.0001$ & $<0.0001$ & 0.01 & 0.01 & 0.003 & 0.001 & 0.14 & 0.86 & 0.01 \\
\hline Interaction & 0.05 & 0.03 & $<0.0001$ & 0.0002 & 0.08 & 0.14 & 0.004 & 0.002 & 0.11 & 0.27 & 0.01 \\
\hline \multicolumn{12}{|c|}{ Marginal means for the two-way ANOVA model } \\
\hline \multicolumn{12}{|c|}{ Saponin supplementation } \\
\hline No saponin & $7 \cdot 8^{\mathrm{a}}$ & $1.9^{\mathrm{b}}$ & $1 \cdot 1^{\mathrm{b}}$ & $2.0^{\mathrm{b}}$ & 1.7 & $3 \cdot 2^{\mathrm{b}}$ & $1.5^{\mathrm{b}}$ & $7 \cdot 9^{\mathrm{a}}$ & $2 \cdot 1$ & $2 \cdot 3$ & $3 \cdot 1^{\mathrm{b}}$ \\
\hline With saponin & $6 \cdot 6^{\mathrm{b}}$ & $3.5^{\mathrm{a}}$ & $2 \cdot 6^{\mathrm{a}}$ & $3.5^{\mathrm{a}}$ & $2 \cdot 3$ & $4 \cdot 2^{a}$ & $3 \cdot 0^{\mathrm{a}}$ & $7 \cdot 1^{\mathrm{b}}$ & $2 \cdot 0$ & 3.1 & $5 \cdot 1^{\mathrm{a}}$ \\
\hline \multicolumn{12}{|l|}{ Basal diet } \\
\hline MG & $7 \cdot 6^{\mathrm{a}, \mathrm{b}}$ & $2 \cdot 7^{\mathrm{b}}$ & $1.3^{\mathrm{b}}$ & $2 \cdot 4^{\mathrm{b}, \mathrm{c}}$ & $2.0^{\mathrm{b}}$ & $3.9^{\mathrm{b}}$ & $2 \cdot 0^{\mathrm{b}, \mathrm{c}}$ & $7 \cdot 6^{\mathrm{a}}$ & 1.5 & 2.4 & $4 \cdot 1^{\mathrm{a}, \mathrm{b}}$ \\
\hline PPC & $5 \cdot 9^{\mathrm{c}}$ & $4 \cdot 8^{\mathrm{a}}$ & $4 \cdot 1^{\mathrm{a}}$ & $5 \cdot 2^{\mathrm{a}}$ & $3 \cdot 2^{\mathrm{a}}$ & $5 \cdot 0^{\mathrm{a}}$ & $3.9^{a}$ & $6 \cdot 2^{\mathrm{b}}$ & 1.7 & $3 \cdot 1$ & $5 \cdot 5^{a}$ \\
\hline SFM & $7 \cdot 8^{\mathrm{a}}$ & $2 \cdot 0^{\mathrm{b}}$ & $1 \cdot 1^{\mathrm{b}}$ & $1 \cdot 7^{\mathrm{c}}$ & $1.6^{\mathrm{b}}$ & $3.3^{\mathrm{b}}$ & $1.7^{\mathrm{b}}$ & $7.9^{a}$ & 1.9 & $2 \cdot 7$ & $3 \cdot 7^{\mathrm{b}, \mathrm{c}}$ \\
\hline RSM & $6 \cdot 6^{\mathrm{b}, \mathrm{c}}$ & $2.4^{\mathrm{b}}$ & $1.7^{\mathrm{b}}$ & $2 \cdot 7^{\mathrm{b}}$ & $1.9^{\mathrm{b}}$ & $3.7^{\mathrm{b}}$ & $2 \cdot 5^{\mathrm{b}, \mathrm{c}}$ & $7 \cdot 7^{\mathrm{a}}$ & 2.9 & $3 \cdot 1$ & $4 \cdot 7^{\mathrm{a}, \mathrm{b}}$ \\
\hline HBM & $8 \cdot 1^{a}$ & $1.5^{\mathrm{b}}$ & $1 \cdot 1^{b}$ & $1 \cdot 6^{c}$ & $1.4^{\mathrm{b}}$ & $2 \cdot 7^{\mathrm{b}}$ & $1 \cdot 2^{\mathrm{C}}$ & $7.9^{\mathrm{a}}$ & $2 \cdot 2$ & 2.5 & $2 \cdot 5^{\mathrm{c}}$ \\
\hline \multicolumn{12}{|c|}{ Means of the diets for the one-way ANOVA model } \\
\hline MG-0 & $7 \cdot 5^{\mathrm{a}}$ & $2 \cdot 4^{\mathrm{b}}$ & $1 \cdot 2^{\mathrm{c}}$ & $2 \cdot 1^{\mathrm{b}, \mathrm{c}}$ & $1.8^{\mathrm{b}}$ & $3 \cdot 4^{\mathrm{c}, \mathrm{d}}$ & $2 \cdot 0^{\mathrm{b}, \mathrm{c}}$ & $7 \cdot 4^{\mathrm{a}}$ & $1.8^{\mathrm{b}, \mathrm{c}}$ & $3 \cdot 0^{\mathrm{a}, \mathrm{b}, \mathrm{c}}$ & $3 \cdot 7^{\mathrm{b}, \mathrm{c}, \mathrm{d}, \mathrm{e}}$ \\
\hline MG-S & $7 \cdot 6^{\mathrm{a}}$ & $3 \cdot 1^{\mathrm{b}}$ & $1 \cdot 5^{\mathrm{b}, \mathrm{c}}$ & $2 \cdot 7^{\mathrm{b}, \mathrm{c}}$ & $2 \cdot 1^{\mathrm{b}}$ & $4 \cdot 4^{\mathrm{b}, \mathrm{c}}$ & $1 \cdot 9^{b, c}$ & $7.9^{\mathrm{a}}$ & $1 \cdot 2^{\mathrm{b}, \mathrm{c}}$ & $1.9^{b, c}$ & $4 \cdot 6^{\mathrm{b}, \mathrm{c}}$ \\
\hline PP-0 & $7.5^{\mathrm{a}}$ & $2 \cdot 4^{\mathrm{b}}$ & $1.4^{\mathrm{b}, \mathrm{c}}$ & $2 \cdot 6^{\mathrm{b}, \mathrm{c}}$ & $2 \cdot 1^{\mathrm{b}}$ & $3 \cdot 7^{\mathrm{c}, \mathrm{d}}$ & $1.4^{\mathrm{c}}$ & $7 \cdot 6^{a}$ & $1 \cdot 6^{\mathrm{b}, \mathrm{c}}$ & $1 \cdot 7^{\mathrm{b}, \mathrm{c}}$ & $3 \cdot 0^{\mathrm{c}, \mathrm{d}, \mathrm{e}}$ \\
\hline PP-S & $4 \cdot 3^{b, c}$ & $7 \cdot 2^{\mathrm{a}}$ & $6 \cdot 8^{\mathrm{a}}$ & $8.0^{\mathrm{a}}$ & $4 \cdot 3^{\mathrm{a}}$ & $6 \cdot 3^{\mathrm{a}}$ & $6 \cdot 5^{\mathrm{a}}$ & $4 \cdot 9^{\mathrm{b}}$ & $1.9^{\mathrm{b}, \mathrm{c}}$ & $4 \cdot 5^{\mathrm{a}}$ & $8.0^{\mathrm{a}}$ \\
\hline SF-0 & $7 \cdot 9^{\mathrm{a}}$ & $1.7^{\mathrm{b}}$ & $0.8^{\mathrm{c}}$ & $1.4^{\mathrm{c}}$ & $1.6^{\mathrm{b}}$ & $3 \cdot 2^{\mathrm{c}, \mathrm{d}}$ & $1 \cdot 3^{\mathrm{c}}$ & $8 \cdot 1^{a}$ & $2 \cdot 8^{\mathrm{a}, \mathrm{b}}$ & $2 \cdot 2^{b, c}$ & $2 \cdot 4^{\mathrm{d}, \mathrm{e}}$ \\
\hline SF-S & $7 \cdot 8^{\mathrm{a}}$ & $2 \cdot 3^{\mathrm{b}}$ & $1 \cdot 3^{\mathrm{b}, \mathrm{c}}$ & $2 \cdot 0^{\mathrm{b}, \mathrm{c}}$ & $1.7^{\mathrm{b}}$ & $3 \cdot 4^{\mathrm{c}, \mathrm{d}}$ & $2 \cdot 2^{b, c}$ & $7 \cdot 8^{a}$ & $1 \cdot 1^{\mathrm{c}}$ & $3 \cdot 1^{a, b, c}$ & $5 \cdot 1^{\mathrm{b}}$ \\
\hline RS-0 & $7 \cdot 4^{\mathrm{a}}$ & $1.5^{\mathrm{b}}$ & $1 \cdot 1^{\mathrm{c}}$ & $2 \cdot 0^{\mathrm{b}, \mathrm{c}}$ & $1.7^{\mathrm{b}}$ & $3 \cdot 2^{\mathrm{c}, \mathrm{d}}$ & $1 \cdot 7^{\mathrm{b}, \mathrm{c}}$ & $8 \cdot 2^{\mathrm{a}}$ & $2 \cdot 2^{\mathrm{a}, \mathrm{b}}$ & $2 \cdot 6^{a, b, c}$ & $4 \cdot 4^{\mathrm{b}, \mathrm{c}, \mathrm{d}}$ \\
\hline RS-S & $5 \cdot 7^{\mathrm{b}}$ & $3 \cdot 3^{\mathrm{b}}$ & $2 \cdot 3^{\mathrm{b}}$ & $3 \cdot 4^{\mathrm{b}}$ & $2 \cdot 2^{\mathrm{b}}$ & $4 \cdot 3^{\mathrm{b}, \mathrm{c}}$ & $3 \cdot 3^{b}$ & $7 \cdot 2^{a}$ & $3 \cdot 6^{\mathrm{a}}$ & $3 \cdot 5^{\mathrm{a}, \mathrm{b}, \mathrm{c}}$ & $5 \cdot 1^{\mathrm{b}}$ \\
\hline HB-O & $8 \cdot 3^{\mathrm{a}}$ & $1.5^{\mathrm{b}}$ & $1 \cdot 1^{\mathrm{C}}$ & $1.7^{\mathrm{c}}$ & $1 \cdot 6^{\mathrm{b}}$ & $2 \cdot 9^{\mathrm{c}, \mathrm{d}}$ & $1.0^{\mathrm{C}}$ & $8 \cdot 1^{a}$ & $2 \cdot 2^{a, b, c}$ & $2 \cdot 1^{b, c}$ & $2 \cdot 1^{\mathrm{e}}$ \\
\hline HB-S & $7 \cdot 7^{\mathrm{a}}$ & $1.6^{\mathrm{b}}$ & $1.1^{\mathrm{c}}$ & $1.5^{\mathrm{c}}$ & $1.1^{\mathrm{b}}$ & $2 \cdot 7^{\mathrm{c}, \mathrm{d}}$ & $1.4^{c}$ & $7 \cdot 8^{\mathrm{a}}$ & $2 \cdot 2^{a, b, c}$ & $2 \cdot 7^{\mathrm{a}, \mathrm{b}, \mathrm{c}}$ & $2 \cdot 9^{\mathrm{c}, \mathrm{d}, \mathrm{e}}$ \\
\hline WG-S & $7 \cdot 4^{\mathrm{a}}$ & $2 \cdot 6^{\mathrm{b}}$ & $1 \cdot 5^{\mathrm{b}, \mathrm{c}}$ & $2 \cdot 4^{\mathrm{b}, \mathrm{c}}$ & $1.0^{\mathrm{b}}$ & $2 \cdot 4^{\mathrm{d}}$ & $2 \cdot 6^{\mathrm{b}, \mathrm{c}}$ & $7 \cdot 3^{\mathrm{a}}$ & $1.5^{\mathrm{b}, \mathrm{c}}$ & $1.4^{\mathrm{c}}$ & $5 \cdot 1^{\mathrm{b}}$ \\
\hline SBM & $3.8^{c}$ & $7 \cdot 0^{\mathrm{a}}$ & $6 \cdot 7^{\mathrm{a}}$ & $7 \cdot 7^{\mathrm{a}}$ & $3.9^{\mathrm{a}}$ & $5 \cdot 8^{a, b}$ & $6 \cdot 6^{a}$ & $5 \cdot 0^{\mathrm{b}}$ & $2 \cdot 0^{\mathrm{b}, \mathrm{c}}$ & $3 \cdot 7^{a, b}$ & $8 \cdot 2^{\mathrm{a}}$ \\
\hline
\end{tabular}

IEL, intra-epithelial lymphocyte; MG, maize gluten; PPC, pea protein concentrate; SFM, sunflower meal; RSM, rapeseed meal; HBM, horsebean meal; -0, non-inclusion; -S, inclusion; PP, pea protein; SF, sunflower; RS, rapeseed; HB, horsebean; WG, wheat gluten; SBM, soyabean meal.

a,b,c,d,e Mean values within a column with unlike superscript letters were significantly different $(P<0.05)$.

* Scores represent means using a visual analogue scale scoring system as described by Penn et al. ${ }^{(20)}$. The score range was arbitrarily set as $0-10$. Scores are based on the actual appearance of each tissue characteristic (i.e. high scores are normal for mucosal fold height and enterocyte vacuolisation; low scores are normal for mucosal fold fusion, lamina propria and submucosa width and cellularity, and enterocyte nucleus position (basal); and intermediate scores are normal for frequency of intraepithelial lymphocytes and goblet cells. 
study, the varying effects of saponin supplementation that depended on basal diets were probably, at least partly, due to varying saponin levels in the diets. However, our chances of evaluating this suggestion are hampered by lack of solid information on saponin content of various plant ingredients, as previously noted. The present diets were analysed for saponins at a well-equipped laboratory, but without reliable results. The different matrices seemed to give different artifacts. We have also made efforts in our own laboratory to analyse sapogenins A and B, the two aglycones of the saponins, with equally inaccurate results. The difficulties seem to be related to variable binding of saponins to the matrices and compounds of the protein sources, as recovery of supplemented saponins differed greatly. It is known that most legumes, peas and horsebeans included, contain similar saponins as soyabeans ${ }^{(33)}$ but in different proportions and at lower levels than that found in soyabeans ${ }^{(26)}$. The saponin content in soyabean seed has been reported at levels from 2 to $60 \mathrm{~g} /$ $\mathrm{kg}^{(34,35)}$. Knudsen et al. ${ }^{(5)}$ suggest that commercial batches of SBM can be expected to contain $5-7 \mathrm{~g} / \mathrm{kg}$. In comparison, the reported content in peas ${ }^{(36-38)}$ varies between 0.7 and $2 \cdot 5 \mathrm{~g} / \mathrm{kg}$. Up-concentration of saponins during pea meal processing, specifically air classification, may be expected. Vicia faba bean varieties differ in their saponin content, with $0.3 \mathrm{~g} / \mathrm{kg}$ reported for broad beans ${ }^{(33)}$ and $0 \cdot 1 \mathrm{~g} / \mathrm{kg}$ for field beans ${ }^{(39)}$, but no reports specifically mention the horsebean variety.

\section{Regarding the basal diets}

The potential of the plant ingredients investigated in the present study as part of multisource feed formulations has been shown for a number of fish species ${ }^{(28,40)}$. In Atlantic salmon, focus is growing and knowledge accumulating on performance of plant ingredients besides soyabeans as sole or multisource formulation replacements of fishmeal as the dietary protein source. However, as mentioned, due to varying levels of fibre and other ANF, the maximum level that can be included in salmonid diets varies greatly.

Previous studies on Atlantic salmon in our group corroborate the good performance observed in the present study with the PPC and HBM basal diets. Aslaksen et al. ${ }^{(16)}$ found that diets with whole peas, whole and dehulled faba beans (closely related to horsebeans) at dietary inclusion rates of 18, 22 and 19\%, respectively, all performed similar to an FM-based control diet. The high retention of digested methionine and cystein for the HBM in the present study was remarkable as the digestibility for these amino acids was also high. Lysine retention was also among the highest for HBM, only second to the value observed for GC, and further strengthens the potential of HBM as a valuable protein source in feed for salmonids. In the study by Aslaksen et $a l^{(16)}$ two pea products produced by air classification with 35\% and 50\% CP content also performed comparably to the FM-based control when fed to Atlantic salmon at $20 \%$ inclusion. However, in a more recent study, we have observed that, as with SBM, a PPC included at 35\% in the diet was associated with negative effects such as reduced growth, reduced lipid digestibility and induction of distal intestinal enteritis in Atlantic salmon ${ }^{(20)}$. In the present study, fish performed well with no signs of the negative effects with an inclusion level of $31 \%$. Thus, the present state of the art indicates that an inclusion level of about $30 \%$ is safe whereas $35 \%$ may be too high to prevent antinutrient-induced consequences, although some products seemingly can be included at $50 \%$ without negative consequences ${ }^{(41)}$. Furthermore, when pea products are combined with other ingredients containing saponins and possibly other antinutrients such as soyabean products, negative nutritional effects may be expected.

Investigations of cereal glutens partially replacing FM in diets of carnivorous farmed fish species such as Atlantic $\operatorname{cod}^{(42)}$, European seabass ${ }^{(43)}$, gilthead seabream ${ }^{(44,45)}$, turbot $^{(46)}$ and rainbow trout ${ }^{(14,47)}$, have shown good performance regarding nutrient utilisation, growth, flesh quality and absence of negative effects on health. WG, replacing up to $50 \%$ of CP from FM, was associated with increased AD of most amino acids except alanine and lysine in diets for Atlantic salmon ${ }^{(15)}$. However, in a study with large $(2.4 \mathrm{~kg}$ initial weight) Atlantic salmon fed a diet incorporating 30\% MG showed lower SGR than fish fed the control FM diet ${ }^{(20)}$. The present results agree with previous investigations in showing values of the basal diet with MG typically falling within the middle range of values observed for other ingredients regarding both nutritional and gut health indicators. In combination, MG and WG supplemented with crystalline amino acids have supported acceptable growth performance and better energy utilisation compared to an FM-based control ${ }^{(48)}$ in feeds for Atlantic salmon. MG and WG, both possess good properties as alternative plant-based protein sources for fish feeds; i.e. low levels of fibre, starch and antinutrients, relatively high protein content with a favourable amino acid profile, high nutrient digestibility and good palatability ${ }^{(40)}$. MG is widely used in fish feeds for salmon ${ }^{(49)}$. Cost of the ingredients has been a major limitation to their use in fish feeds, but sharp increases in the relative price of fishmeal will eventually make these refined products increasingly more economical to use ${ }^{(40)}$.

The comparatively low performance of the SFM basal diet may be related to the SFM content of ANF such as a high fibre content, oligosaccharides, phytic acid and arginase inhibitor $^{(27)}$. This is somewhat in contrast to the general consideration that SFM is a valuable protein source with good availability and relatively few ANF. Dehulling and extrusion nutritionally improve the ingredient as shown by Gill et al. $^{(50)}$ who recommended SFM use in Atlantic salmon feed formulations at inclusion levels of up to $23 \%$ of digestible dietary protein based on results showing no adverse effects on fish performance. However, in another study, and in line with the present results, feeding extruded diets incorporating $20 \%$ CP from SFM to Atlantic salmon reduced protein digestibility $^{(16)}$. Other possibly beneficial dietary effects of SFM have been observed associated with reduced sea lice infestation on Atlantic salmon ${ }^{(51)}$. Further studies on the nutritional and health aspects of SFM are, therefore, needed for better understanding of the effects of SFM in diets for Atlantic salmon and other farmed fish species. 
In the present study, the lowest fish performance was associated with the RSM basal diet. This finding was in agreement with the low performance of RSM diets also observed by Aslaksen et $a l .{ }^{(16)}$. This poor performance can be attributed to the presence of heat-stable glucosinolates, high fibre and a potent trypsin inhibitor ${ }^{(27,28)}$. Removal of antinutrients seems necessary for RS to become a useful protein source for Atlantic salmon.

\section{Conclusions}

Dietary soyasaponin supplementation at a level of $0 \cdot 2 \%$, a level expected in diets with approximately 20-30\% SBM, caused minor to moderate effects on fish growth, nutrient utilisation and intestinal physiology when supplemented to diets with MG, SFM, HBM and RSM. In the diet with PPC, the saponin supplementation induced major effects that were similar to the typical effects induced by SBM diets. Our present study therefore confirms earlier suggestions that saponins are contributors to the development of diet-induced enteritis in salmonids as seen with SBM and high levels of PPC. Care should be taken when combining plant ingredients containing saponins such as peas and SBM and possibly also other saponincontaining ingredients to avoid high levels of saponins.

\section{Acknowledgements}

The present work was carried out under an industry-driven project led by BioMar AS and partly funded by The Research Council of Norway (project no. 187294) and the Aquaculture Protein Centre (project no. 145949/120). There were no conflicts of interest for any of the authors involved in this work. The authors' contributions were as follows: E. M. C. contributed in the areas of experimental design, sampling, statistics and manuscript write-up; F. F. V. contributed to the experimental design, sampling, sample analyses and manuscript write-up; M. H. P., to the experimental design, sampling, histology and manuscript review; J. R., to the experimental design, sampling and manuscript review; S. R., to the experimental design, feeding and husbandry of experimental fish, sampling and manuscript review; A. G., to the experimental design, feed production, sampling and manuscript review; M. H., to the experimental design, feed formulation and production, sampling and manuscript review; andÅ. K. contributed to the experimental design, sampling, statistics, manuscript write-up and review. Thanks are due to the animal technicians at Nofima Marin at Sunndalsøra for excellent fish care and management and to the laboratory technicians at Nofima Marin and the Gut and Health Group of the Aquaculture Protein Centre for skilful performance of all the necessary analyses.

\section{References}

1. van den Ingh T, Krogdahl $\AA$, Olli JJ, et al. (1991) Effects of soybean-containing diets on the proximal and distal intestine in Atlantic salmon (Salmo salar): a morphological study. Aquaculture 94, 297-305.
2. Olli JJ, Krogdahl A, Ingh T, et al. (1994) Nutritive value of four soybean products in diets for Atlantic salmon. Acta Agr Scand Sect A: Anim Sci 44, 50-60.

3. Olli JJ \& Krogdahl A (1995) Dehulled solvent-extracted soybean meal as a protein source in diets for Atlantic salmon Salmo salar L.. Aquaculture Res 26, 167-174.

4. Uran PA, Goncalves AA, Taverne-Thiele JJ, et al. (2008) Soybean meal induces intestinal inflammation in common carp (Cyprinus carpio L.). Fish Shellfish Immunol 25, 751-760.

5. Knudsen D, Ron O, Baardsen G, et al. (2006) Soyasaponins resist extrusion cooking and are not degraded during gut passage in Atlantic salmon (Salmo salar L.). J Agric Food Chem 54, 6428-6435.

6. Knudsen D, Uran P, Arnous A, et al. (2007) Saponin-containing subfractions of soybean molasses induce enteritis in the distal intestine of Atlantic salmon. I Agric Food Chem 55, 2261-2267.

7. Knudsen D, Jutfelt F, Sundh H, et al. (2008) Dietary soya saponins increase gut permeability and play a key role in the onset of soyabean-induced enteritis in Atlantic salmon (Salmo salar L.). Br J Nutr 100, 120

8. Shi J, Arunasalam K, Yeung D, et al. (2004) Saponins from edible legumes: chemistry, processing, and health benefits. J Med Food 7, 67-78.

9. Krogdahl $\AA$, Penn M, Thorsen J, et al. (2010) Important antinutrients in plant feedstuffs for aquaculture: an update on recent findings regarding responses in salmonids. Aquacult Res 41, 333-344.

10. Johnson IT, Gee JM, Price K, et al. (1986) Influence of saponins on gut permeability and active nutrient transport in vitro. J Nutr 116, 2270-2277.

11. Francis G, Kerem Z, Makkar HP, et al. (2002) The biological action of saponins in animal systems: a review. Br J Nutr $\mathbf{8 8}$ 587-605.

12. Bureau DP, Harris AM \& Young Cho C (1998) The effects of purified alcohol extracts from soy products on feed intake and growth of chinook salmon (Oncorbynchus tshawytscha) and rainbow trout (Oncorbynchus mykiss). Aquaculture 161, 27-43.

13. Francis G, Makkar HPS \& Becker K (2005) Quillaja saponins - a natural growth promoter for fish. Anim Feed Sci Technol 121, $147-157$.

14. Davies SJ, Morris PC \& Baker RTM (1997) Partial substitution of fish meal and full-fat soya bean meal with wheat gluten and influence of lysine supplementation in diets for rainbow trout, Oncorbynchus mykiss (Walbuam). Aquacult Res 28, $317-328$

15. Storebakken T, Shearer KD, Baeverfjord G, et al. (2000) Digestibility of macronutrients, energy, and amino acids, absorption of elements and absence of intestinal enteritis in Atlantic salmon, Salmo salar, fed diets with wheat gluten. Aquaculture 184, 115-132.

16. Aslaksen MA, Kraugerud OF, Penn M, et al. (2007) Screening of nutrient digestibilities and intestinal pathologies in Atlantic salmon, Salmo salar, fed diets with legumes, oilseeds, or cereals. Aquaculture 272, 541-555.

17. Refstie S, Helland SJ \& Storebakken T (1997) Adaptation to soybean meal in diets for rainbow trout, Oncorbynchus mykiss. Aquaculture 153, 263-272.

18. Kakade ML, Hoffa DE \& Liener IE (1973) Contribution of trypsin inhibitors to the deleterious effects of unheated soybeans fed to rats. $J$ Nutr 103, 1772-1778.

19. Krogdahl ^̊, Bakke-McKellep AM \& Baeverfjord G (2003) Effects of graded levels of standard soybean meal on intestinal structure, mucosal enzyme activities, and pancreatic 
response in Atlantic salmon (Salmo salar L.). Aquacult Nutr 9, 361-371.

20. Penn MH, Bendiksen E̊, Campbell P, et al. (2010) High level of dietary pea protein concentrate induces enteropathy in Atlantic salmon (Salmo salar L.). Aquaculture 310, 267-273.

21. Helland SJ, Grisdale-Helland B \& Nerland S (1996) A simple method for the measurement of daily feed intake of groups of fish in tanks. Aquaculture 139, 157-163.

22. Austreng E, Storebakken T, Thomassen MS, et al. (2000) Evaluation of selected trivalent metal oxides as inert markers used to estimate apparent digestibility in salmonids. Aquaculture 188, 65-78.

23. Messina MJ (1999) Legumes and soybeans: overview of their nutritional profiles and health effects. Am J Clin Nutr $\mathbf{7 0}$ (Suppl. 3), 439-450.

24. Twibell RG \& Wilson RP (2004) Preliminary evidence that cholesterol improves growth and feed intake of soybean meal-based diets in aquaria studies with juvenile channel catfish, Ictalurus punctatus. Aquaculture 236, 539-546.

25. Wang N \& Daun JK (2004) Effect of variety and crude protein content on nutrients and certain antinutrients in field peas (Pisum sativum). J Sci Food Agric 84, 1021-1029.

26. Vidal-Valverde C, Frias J, Hernandez A, et al. (2003) Assessment of nutritional compounds and antinutritional factors in pea (Pisum sativum) seeds. J Sci Food Agric 83, 298-306.

27. Francis G, Makkar HPS \& Becker K (2001) Antinutritional factors present in plant-derived alternate fish feed ingredients and their effects in fish. Aquaculture 199, 197-227.

28. Drew MD, Borgeson TL \& Thiessen DL (2007) A review of processing of feed ingredients to enhance diet digestibility in finfish. Anim Feed Technol 138, 118-136.

29. Eriksson S, Andréasson E, Ekbom B, et al. (2002) Complex formation of myrosinase isoenzymes in oilseed rape seeds are dependent on the presence of myrosinase-binding proteins. Plant Physiol 129, 1592-1599.

30. Grosell M (2006) Intestinal anion exchange in marine fish osmoregulation. J Exp Biol 209, 2813-2827.

31. Gee JM, Wortley GM, Johnson IT, et al. (1996) Effects of saponins and glycoalkaloids on the permeability and viability of mammalian intestinal cells and on the integrity of tissue preparations in vitro. Toxicol In vitro 10, 117-128.

32. Chikwati EM (2007) Effects of soyasaponins, phytosterols, chitosan and Orlistat on digestive function and histomorphology of the intestinal tract of Atlantic salmon (Salmo salar L.). Master degree thesis. Norwegian School of Veterinary Science.

33. Kinjo J, Hatakeyama M, Udayama M, et al. (1998) HPLC profile analysis of oleanene-glucuronides in several edible beans. Biosci Biotechnol Biochem 62, 429-433.

34. Shiraiwa M, Harada K, Okubo K, et al. (1991) Composition and content of saponins in soybean seed according to variety, cultivation year and maturity. Agric Biol Chem 55, 323-331.

35. Anderson RL \& Wolf WJ (1995) Compositional changes in trypsin inhibitors, phytic acid, saponins and isoflavones related to soybean processing. J Nutr $\mathbf{1 2 5}, 581 \mathrm{~S}-588 \mathrm{~S}$.

36. Champ MM (2002) Non-nutrient bioactive substances of pulses. Br J Nutr 88, S307-S319.
37. Daveby YD, Aman P, Betz JM, et al. (1997) The variation in content and changes during development of Soyasaponin I in dehulled Swedish peas (Pisum sativum L.). J Sci Food Agric 73, 391-395.

38. Heng L, Vincken JP, van Koningsveld G, et al. (2006) Bitterness of saponins and their content in dry peas. $J$ Sci Food Agric 86, 1225-1231.

39. Price KR, Curl CL \& Fenwick GR (1986) The saponin content and sapogenol composition of the seed of 13 varieties of legume. J Sci Food Agric 37, 1185-1191.

40. Naylor RL, Hardy RW, Bureau DP, et al. (2009) Feeding aquaculture in an era of finite resources. Proc Natl Acad Sci U S A 106, 15103-15110.

41. Øverland M, Sørensen M, Storebakken T, et al. (2009) Pea protein concentrate substituting fish meal or soybean meal in diets for Atlantic salmon (Salmo salar) - effect on growth performance, nutrient digestibility, carcass composition, gut health, and physical feed quality. Aquaculture 288, 305-311.

42. Tibbetts SM, Milley JE \& Lall S (2006) Apparent protein and energy digestibility of common and alternative feed ingredients by Atlantic cod, Gadus morbua (Linnaeus, 1758). Aquaculture 261, 1314-1327.

43. Robaina L, Corraze G, Aguirre P, et al. (1999) Digestibility, postprandial ammonia excretion and selected plasma metabolites in European sea bass (Dicentrarchus labrax) fed pelleted or extruded diets with or without wheat gluten. Aquaculture 179, 45-56.

44. Robaina L, Moyano FJ, Izquierdo MS, et al. (1997) Corn gluten and meat and bone meals as protein sources in diets for gilthead seabream (Sparus aurata): nutritional and histological implications. Aquaculture 157, 347-359.

45. Pereira TG \& Oliva-Teles A (2003) Evaluation of corn gluten meal as a protein source in diets for gilthead seabream (Sparus aurata L.) juveniles. Aquacult Res 34, 1111-1117.

46. Sugiura SH, Dong FM, Rathbone CK, et al. (1998) Apparent protein digestibility and mineral availabilities in various feed ingredients for salmonid feeds. Aquaculture 159, $177-202$.

47. Skonberg DI, Hardy RW, Barrows FT, et al. (1998) Color and flavor analyses of fillets from farm-raised rainbow trout (Oncorbynchus mykiss) fed low-phosphorus feeds containing corn or wheat gluten. Aquaculture 166, 269-277.

48. Espe M, Lemme A, Petri A, et al. (2006) Can Atlantic salmon (Salmo salar) grow on diets devoid of fish meal? Aquaculture 255, 255-262.

49. Gatlin DM III, Barrows FT, Brown P, et al. (2007) Expanding the utilization of sustainable plant products in aquafeeds: a review. Aquacult Res 38, 551-579.

50. Gill N, Higgs DA, Skura BJ, et al. (2006) Nutritive value of partially dehulled and extruded sunflower meal for postsmolt Atlantic salmon (Salmo salar L.) in seawater. Aquacult Res 37, 1348-1359.

51. Refstie S, Baeverfjord G, Seim RR, et al. (2010) Effects of dietary yeast cell wall $\beta$-glucans and MOS on performance, gut health, and salmon lice resistance in Atlantic salmon (Salmo salar) fed sunflower and soybean meal. Aquaculture 305, 109-116. 